\title{
VEGETACIÓN Y FLORA DE LA REGIÓN DE NIZANDA, ISTMO DE TEHUANTEPEC, OAXACA, MÉXICO
}

\author{
Eduardo A. Pérez-García \\ Jorge MeAve \\ Y \\ Claudia Gallardo
}
Laboratorio de Ecología, Departamento de Ecología y Recursos Naturales Facultad de Ciencias, Universidad Nacional Autónoma de México, 04510 México, D.F. correo electrónico: eapg@hp.fciencias.unam.mx

\begin{abstract}
RESUMEN
En la región de Nizanda, área botánicamente poco conocida del Istmo de Tehuantepec, Distrito de Juchitán (Oaxaca), se realizó una caracterización general de las principales comunidades vegetales y se elaboró un inventario de la flora basado en colectas originales. La heterogeneidad del paisaje determina la existencia de siete tipos de vegetación: bosque de galería, matorral espinoso, matorral xerófilo, sabana, selva baja caducifolia, selva mediana (con dos variantes, subcaducifolia y subperennifolia), y vegetación acuática y subacuática. La lista florística incluye 746 especies, 458 géneros y 119 familias de plantas vasculares. La información de las 1,435 colectas realizadas fue incorporada a una base de datos relacional, lo cual permitió especificar la distribución de las especies en los tipos de vegetación. Se discuten de manera general los patrones de la vegetación, sus posibles causas y la importancia de la flora de Nizanda a nivel regional.
\end{abstract}

Palabras clave: Comunidades vegetales, flora, Nizanda, Oaxaca, México.

\begin{abstract}
In the Nizanda region, a botanically little known area located in the Isthmus of Tehuantepec, Juchitan District, Oaxaca (southern Mexico), the main plant communities were characterized, and a floristic inventory was prepared on the basis of our own collected material. Landscape heterogeneity in Nizanda determines the existence of seven vegetation types: gallery forest, thorn scrub, xeric scrub, savanna, dry tropical forest, semievergreen and semideciduous tropical forest, and aquatic and subaquatic vegetation. The floristic list includes a total of 746 species of vascular plants, distributed in 458 genera and 119 families. The 1,435 collection records were fed into a relational database, which allowed us to determine for each species the vegetation types where it was found. General vegetation patterns are discussed, together with their possible causes, as well as the relevance of the flora of Nizanda at the regional level.
\end{abstract}

Key words: Vegetation community, flora, Nizanda, Oaxaca, Mexico. 


\section{INTRODUCCIÓN}

Diversas características hacen del Istmo de Tehuantepec una de las áreas más interesantes de México desde el punto de vista biológico. Su condición de istmo le confiere el doble papel de puente entre las vertientes atlántica y pacífica de México, situación sólo compartida en el resto de Mesoamérica con la Depresión de Nicaragua y el istmo panameño, y de barrera biogeográfica para las biotas montanas, incapaces de atravesar las tierras bajas (Wendt, 1998; Peterson et al., 1999). Asimismo, su ubicación geográfica es privilegiada por estar situada prácticamente en la zona de contacto de los reinos biogeográficos Neotropical y Neoártico. Además, el Istmo también posee un alto nivel de endemismo (Lorence y GarcíaMendoza, 1989). A pesar de que estos factores han promovido una gran diversidad biológica en la comarca istmeña, es notable la escasez de esfuerzos sistemáticos para conocerla. La paradoja que representa la alta diversidad y el desconocimiento biológico del Istmo de Tehuantepec es particularmente cierta en el caso de la flora, a pesar de las numerosas visitas de naturalistas y botánicos a la región en los últimos tres siglos. Entre los pioneros destacan M. Mociño en el siglo XVIII y G. Andrieux en el XIX (Torres et al., 1997). En la primera mitad del siglo XX, T. MacDougall realizó importantes colectas en la década de 1930, y más tarde Williams (1939) publicó "Árboles y arbustos del Istmo de Tehuantepec", primera obra que trata de la flora de esta región. Posteriormente, en su inventario biológico nacional, Goldman (1951) citó colectas de las cercanías de Nizanda, localidad donde se centró el presente estudio, y Duellman (1960) describió la vegetación de las crestas centrales del Istmo de Tehuantepec, elevaciones que definen el parteaguas continental y marcan la transición de la región húmeda del Golfo de México hacia la subhúmeda del Pacífico.

Más recientemente se han publicado descripciones de floras locales en la región del Istmo de Tehuantepec. Zizumbo y Colunga (1980) elaboraron una lista florística de la zona huave de San Mateo del Mar, y Torres-Colín (1989) publicó el listado de plantas del cerro Guiengola. El listado florístico más completo que existe para la región istmeña es el de Torres-Colín et al. (1997) del Distrito de Tehuantepec, el cual incluye 1,720 especies recolectadas en doce tipos de vegetación. Sin embargo, estos esfuerzos por incrementar el conocimiento botánico de la región han sido insuficientes, lo cual se refleja en la existencia de grandes porciones del Istmo que permanecen inexploradas y en el estado taxonómico incierto de muchas especies que allí habitan.

Una de las áreas menos estudiadas de la vertiente pacífica del Istmo, pero posiblemente una de las más interesantes, es la conocida como el "Paso de Chivela", localizada justo en los límites de las regiones centrales y la Planicie Costera de Tehuantepec. En ella es notoria la transición entre el clima cálido húmedo del Golfo de México al tropical estacional característico de la vertiente mexicana del Pacífico. La región de Nizanda, ubicada en dicha porción del Istmo, alberga muchas plantas que han sido utilizadas para la tipificación y denominación de varias especies y subespecies; entre ellas se pueden citar a Agave nizandensis (Cutak, 1951), Anthurium nizandense (Matuda, 1959), Barkeria whartoniana (Soto-Arenas, 1993) y Cephalocereus nizandensis (Bravo y MacDougall, 1958).

La carencia de información botánica referente a la región de Nizanda, y los indicios de que en ella habita un conjunto de plantas interesantes, motivaron la realización del 
presente estudio, cuyos objetivos fueron: (1) caracterizar de manera general las principales comunidades vegetales de la región de Nizanda, y (2) realizar un inventario de la flora de esta zona, basado en colectas originales. En la lista florística que resultó de este estudio se hace un desglose de los diferentes tipos de vegetación donde fueron recolectadas las especies, con el fin de ubicarlas espacialmente en el complejo mosaico de comunidades bióticas presentes en la región.

\section{DESCRIPCIÓN DEL ÁREA DE ESTUDIO}

\section{Localización}

El estudio se llevó a cabo en la región centrada en una población cuyo nombre oficial es Mena, pero que es mejor conocida como Nizanda por sus pobladores. Nizanda se localiza a orillas del ferrocarril transístmico, a $13.5 \mathrm{~km}$ al norte de Ciudad Ixtepec $\left(16^{\circ} 39^{\prime} \mathrm{N}, 95^{\circ} 00^{\prime} \mathrm{W}\right)$. Aunque el poblado pertenece al municipio de Asunción Ixtaltepec, una parte de la región de estudio está ubicada en el municipio de Ciudad Ixtepec, ambos pertenecientes al Distrito de Juchitán, Oaxaca.

La región de Nizanda cubre aproximadamente $85 \mathrm{~km}^{2}$ y se define de manera arbitraria como la porción del Istmo de Tehuantepec limitada al sur por la Planicie Costera de Tehuantepec; al norte por las planicies de Chivela, ubicadas al norte del parteaguas continental; al oeste por las laderas orientales del Cerro Naranjo; y al este por el tramo Chivela - La Ventosa de la carretera federal No. 185 (Fig. 1).

\section{Geología y edafología}

En la región dominan rocas del Mesozoico, particularmente complejos metamórficos (esquistos) y rocas calizas del Cretácico Inferior (Anónimo, 1981a). Los suelos predominantes en la región istmeña son Litosoles, aunque en extensiones menores se presentan también Feozems háplicos y Regosoles éutricos, ambos de textura media. Hacia el sur de Nizanda, en la Planicie Costera de Tehuantepec y en el valle del Río Verde, el suelo prevaleciente es Cambisol éutrico y, en menor proporción, Cambisol crómico de textura media (Anónimo, 1981a).

En las cimas de las lomas de esquistos los suelos son someros y poseen muchas gravas superficiales, pero hacia el piedemonte están más desarrollados. En las planicies aluviales los suelos son más profundos $(>1 \mathrm{~m}$ ) y arenosos, aunque en ocasiones presentan texturas más finas. Sobre las laderas de los cerros calizos el gradiente edáfico es más acentuado, ya que abarca desde suelos con profundidades $>50 \mathrm{~cm}$, de colores rojizos en sus horizontes inferiores, con una estructura marcada y una notoria acumulación de arcillas en el piedemonte, hasta una somera acumulación de materia orgánica entre las grietas de la roca expuesta en las cimas. 

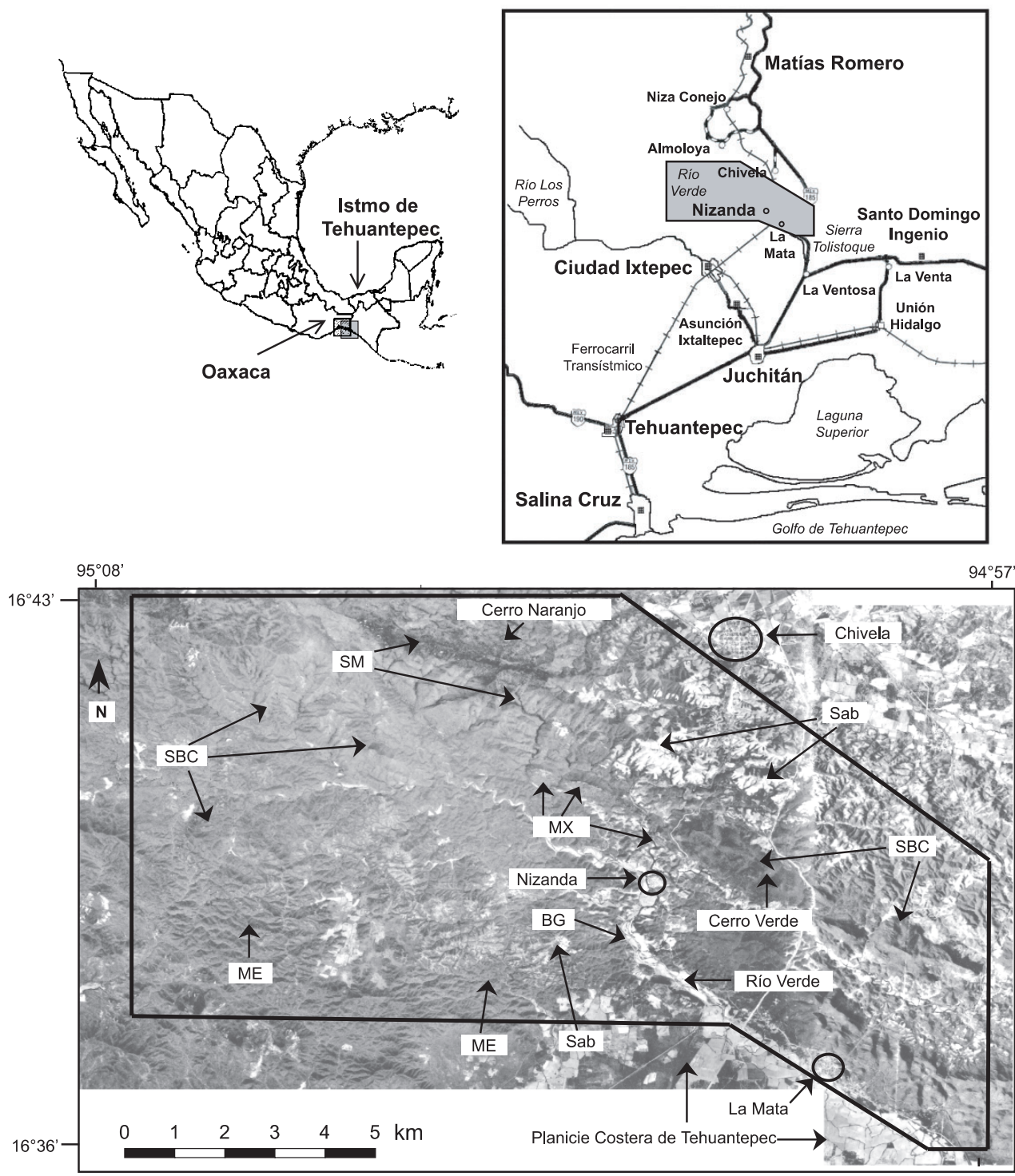

Fig. 1. (Parte superior). Localización de la región de Nizanda en Oaxaca y de la porción sur del Istmo de Tehuantepec. (Parte inferior). Fotocomposición aérea de la región de Nizanda basada en fotografías escala 1:75,000 (INEGI, marzo de 1991). Se muestran los límites de la región estudiada y algunos sitios selectos donde se presentan los tipos de vegetación descritos en el texto que son distinguibles a esta escala: $\mathrm{BG}$ = bosque de galería; $\mathrm{ME}$ = matorral espinoso; $\mathrm{MX}$ = matorral xerófilo; $\mathrm{Sab}=$ sabana; $\mathrm{SBC}=$ selva baja caducifolia; $\mathrm{SM}=$ selva mediana . 
Relieve

Alrededor de Nizanda confluyen varios sistemas montañosos y esta situación dificulta definir con exactitud el origen de los cerros que conforman este intrincado nudo de sierras. En términos generales se puede decir que la región está bordeada al oeste y suroeste por la Sierra Madre del Sur (Sierra de Miahuatlán), al noroeste por la Sierra Norte de Oaxaca (Sierra Mixe), al este por la Sierra Tolistoque, y al sur por los terrenos Ilanos de la Planicie Costera del Golfo de Tehuantepec.

La geomorfología dominante consiste en lomeríos de baja altitud (cerca de $250 \mathrm{~m}$ s.n.m.), con laderas medianamente pronunciadas, moldeadas principalmente por erosión hídrica. Este paisaje está interrumpido de manera abrupta por la presencia de cerros muy escarpados formados por afloramientos de roca caliza; el Cerro Verde, que es el más alto de ellos, alcanza una elevación aproximada de $500 \mathrm{~m}$. Las laderas de los cerros kársticos son muy inclinadas, y en muchas porciones existen escarpes casi verticales. La altitud de la zona varía desde poco menos de 100 m s.n.m. en el cauce bajo del Río Verde, a más de 700 m s.n.m. en la cima del Cerro Naranjo (Anónimo 1981b, 1988a, b).

Clima

Aunque en Nizanda no existe una estación meteorológica, la información proveniente de estaciones aledañas (Cuadro 1) permite deducir una temperatura promedio para Nizanda de alrededor de $25^{\circ} \mathrm{C}$ y una precipitación promedio anual de aproximadamente $1,000 \mathrm{~mm}$. Las lluvias caen con una marcada estacionalidad, distinguiéndose una época seca de noviembre a abril, y una lluviosa de mayo a octubre (Anónimo, 1984a, b). Es probable que el clima de Nizanda sea similar al registrado en Ciudad Ixtepec ( $\left.A w_{0}(w) i g w "\right)$, es decir, cálido subhúmedo con lluvias en verano (García, 1988).

A pesar de su situación cerca de la trayectoria normal de los ciclones tropicales del Pacífico nororiental, la incidencia de estos fenómenos en la región es poco frecuente (Jáuregui, 1984). Por el contrario, la aceleración de los vientos alisios provenientes del Golfo de México, que encuentran en el Istmo de Tehuantepec el único paso interoceánico de baja altitud en nuestro país (Chelton et al., sin fecha), hace que la región reciba casi continuamente el impacto de vientos de gran velocidad, los cuales son particularmente fuertes entre septiembre y mayo (Rodrigo Álvarez, 1994).

Hidrología

En Nizanda convergen tres corrientes de agua menores: proveniente del norte corre el arroyo Agua Tibia, llamado así por estar alimentado permanentemente por un manantial termal; desde el este fluye el arroyo Verde (también conocido como Mazahua); y por el oeste se incorpora el arroyo Chilona. Estos dos últimos son corrientes estacionales. De su unión se forma el Río Verde, el cual fluye hacia la Planicie Costera del Golfo de Tehuantepec. Nizanda significa en zapoteco "agua que nace caliente". Este vocablo hace alusión a sus afloramientos de agua hipogea termal, los cuales permiten el establecimiento de comunidades vegetales con requerimientos hídricos más elevados. 
Cuadro 1. Ubicación de estaciones meteorológicas e información climática más relevante de éstas a lo largo del Istmo de Tehuantepec (García, 1988). Las estaciones están ordenadas en sentido nortesur. Los valores para Nizanda marcados con un asterisco son estimaciones y la fórmula climática para esta localidad es la más probable.

\begin{tabular}{|c|c|c|c|c|c|c|}
\hline \multirow[t]{2}{*}{ Estación } & \multirow{2}{*}{$\begin{array}{c}\text { Altitud } \\
\text { (m s.n.m.) }\end{array}$} & \multicolumn{2}{|c|}{ Localización } & \multirow{2}{*}{$\begin{array}{c}\text { Precipitación } \\
\text { anual }(\mathrm{mm})\end{array}$} & \multirow{2}{*}{$\begin{array}{c}\text { Temperatura } \\
\text { media anual } \\
\left({ }^{\circ} \mathrm{C}\right)\end{array}$} & \multirow{2}{*}{$\begin{array}{l}\text { Fórmula } \\
\text { climática }\end{array}$} \\
\hline & & Lat. N & Long. $\mathrm{O}$ & & & \\
\hline Coatzacoalcos & 14 & $18^{\circ} 09^{\prime}$ & $94^{\circ} 25^{\prime}$ & 2780.1 & 25.4 & $A m\left(i^{\prime}\right) g w^{\prime \prime}$ \\
\hline Acayucan & 88 & $17^{\circ} 57^{\prime}$ & $95^{\circ} 55^{\prime}$ & 1771.6 & 25.5 & $A w_{2}\left(i^{\prime}\right) g w^{\prime \prime}$ \\
\hline Sarabia & 80 & $17^{\circ} 05^{\prime}$ & $95^{\circ} 03^{\prime}$ & 2384.2 & 25.1 & $\mathrm{Am}(\mathrm{e}) \mathrm{g}$ \\
\hline Matías Romero & 201 & $16^{\circ} 53^{\prime}$ & $95^{\circ} 03^{\prime}$ & 1432.6 & 24.7 & $A w_{2}(w) i g w "$ \\
\hline Chivela & 180 & $16^{\circ} 43^{\prime}$ & $95^{\circ} 00^{\prime}$ & 1194.2 & 24.8 & $A w_{1}(w)\left(i^{\prime}\right) g w^{\prime \prime}$ \\
\hline Nizanda & $90-120$ & $16^{\circ} 39^{\prime}$ & $95^{\circ} 00^{\prime}$ & $1000-1100^{*}$ & $25.0^{*}$ & $A w_{0}(w) i g w "$ \\
\hline Cd. Ixtepec & $50^{+}$ & $16^{\circ} 34^{\prime}$ & $95^{\circ} 06^{\prime}$ & 880.5 & 27.5 & $A w_{0}(w) i g w "$ \\
\hline Juchitán & 46 & $16^{\circ} 26^{\prime}$ & $95^{\circ} 02^{\prime}$ & 928.7 & 27.3 & $A w_{0}(\mathrm{w})$ igw" $^{\prime}$ \\
\hline Tehuantepec & 55 & $16^{\circ} 19^{\prime}$ & $95^{\circ} 13^{\prime}$ & 874.9 & 28.2 & $A w_{0}(w) i g w "$ \\
\hline Salina Cruz & 6 & $16^{\circ} 11^{\prime}$ & $95^{\circ} 11^{\prime}$ & 1058.4 & 27.4 & $A w_{0}(w) i g w "$ \\
\hline
\end{tabular}

† En la obra de García (1988) se cita una altitud de 1,200 m s.n.m. para esta localidad, pero ese dato es erróneo.

\section{MÉTODO}

El trabajo se sustenta en las colectas realizadas en la zona, efectuadas entre diciembre de 1995 y septiembre de 2000. Éstas no incluyeron a las plantas cultivadas ni a las de ornato que se encuentran en los poblados y sus inmediaciones, sino que se centraron en las silvestres.

El primer juego de los ejemplares está depositado en el Herbario Nacional (MEXU) de la Universidad Nacional Autónoma de México. Duplicados de estos especímenes están depositados en los herbarios OAX y FCME. Los registros de las colectas se capturaron en el modelo "Biótica" para una base de datos relacional desarrollada por la Comisión Nacional para el Conocimiento y Uso de la Biodiversidad (versión para ACCESS 97), la cual puede consultarse a través de la Dirección de Servicios Externos de dicha institución (www.conabio.gob.mx). Este procedimiento permitió elaborar la lista florística y definir la distribución de las especies por tipo de vegetación. En la determinación de ejemplares participaron numerosos especialistas, cuyos nombres se mencionan en los Agradecimientos.

Los tipos de vegetación de Nizanda fueron diferenciados con base en atributos fisonómicos y fenológicos. La nomenclatura utilizada combina criterios de las clasificaciones de Miranda y Hernández-X. (1963) y de Rzedowski (1978), pero incorpora algunas consideraciones personales (Pérez-García et al., 1998b), las cuales se indican en la sección de resultados. La mayoría de los tipos de vegetación corresponden al sistema de Miranda y Hernández-X., particularmente por el uso del término selva. De Rzedowski (1978) se utilizan las categorías matorral xerófilo y bosque de galería, aunque el uso de este último 
término se restringió a la vegetación arbórea ribereña poco diversa. La elección de las especies que acompañan las descripciones de las comunidades vegetales se basó en observaciones de campo y en resultados de los muestreos de vegetación realizados en la zona.

El reconocimiento de las familias de Pteridophyta se basó en Moran y Riba (1995); para las de Magnoliopsida se utilizó el sistema propuesto por Cronquist (1981), mientras que para las Liliopsida se siguió el de Dahlgren et al. (1985). El listado tiene el siguiente orden por secciones: 1) Pteridophyta, 2) Cycadophyta, 3) Magnoliopsida y 4) Liliopsida. En cada sección, las familias y las especies se ordenaron alfabéticamente. Los autores de los taxa se abrevian de acuerdo con Brummitt y Powell (1992). La nomenclatura usada por Torres-Colín et al. (1997) sirvió como base para la denominación de los géneros y las especies, pero en general se respetaron las determinaciones de los especialistas. De igual manera, ese trabajo se utilizó como una primera evaluación del conocimiento de la flora regional (ya que no existe algo similar para el Distrito de Juchitán) y por ello, en la lista que aparece en el Apéndice, se indican con un asterisco las especies que no fueron incluidas en dicha obra. Lo anterior no significa necesariamente que éstas califiquen como nuevos registros para la región; más bien, se deben interpretar como una medida de la divergencia florística entre áreas geográficas adyacentes.

\section{RESULTADOS}

Flora

La lista de especies incluye un total de 746 especies que representan a 458 géneros pertenecientes a 119 familias (Apéndice). La base de datos contiene 1,435 registros, correspondientes a igual número de colectas que sustentan esta información. Tales datos, junto con los ejemplares recolectados que no han sido determinados y las morfoespecies distinguidas a partir de individuos estériles que se han encontrado en los muestreos de vegetación, permiten calcular de manera conservadora que el número total de especies presentes en la región debe ubicarse entre 850 y 900.

Casi 39\% (287) de las especies de la lista aquí presentada no estaban previamente registradas en el trabajo de Torres-Colín et al. (1997). Si bien es necesario tomar esta cifra con reserva, en virtud de las posibles discordancias en las determinaciones, es indudable que se trata de una cifra importante, considerando el reducido tamaño del área trabajada y su cercanía al Distrito de Tehuantepec.

Las familias más ricas en especies de la flora de Nizanda son Fabaceae (57 especies), Poaceae (52), Asteraceae (44), Mimosaceae (33), Euphorbiaceae (39), Orchidaceae (24), Caesalpiniaceae (23), Convolvulaceae (21), Rubiaceae (19), Bignoniaceae y Cactaceae (18 cada una). La mayoría de estas familias son las que presentan la mayor riqueza genérica: Asteraceae (36 géneros), Fabaceae (29), Poaceae (20), Orchidaceae (19), Cactaceae (16), Mimosaceae (14), Bignoniaceae y Rubiaceae (13 cada una), y Euphorbiaceae y Malpighiaceae (11 cada una). Las tres familias que conforman el grupo de las leguminosas abarcan en conjunto 113 especies y 51 géneros. 
Entre los géneros que destacan por su riqueza específica están: Ipomoea (12), Mimosa (9), Acacia, Croton, Cyperus y Tillandsia (8 cada uno), Dioscorea y Euphorbia (7 cada uno), Chamaecrista, Cordia, Ficus y Senna (6 cada uno). El cociente especies/géneros tuvo sus valores más bajos en las familias Cactaceae (1.13), Rhamnaceae y Verbenaceae (1.20), Apocynaceae y Asteraceae (1.22), Orchidaceae (1.26), Acanthaceae (1.29), Bignoniaceae (1.38), Rutaceae (1.40) y Malpighiaceae (1.45). En contraste, los valores más altos para este cociente correspondieron a familias con pocas especies y un solo género: Dioscoreaceae (7.0), y Burseraceae, Passifloraceae y Polygalaceae (5.0 cada una). Las Caesalpiniaceae representan una excepción notable dentro de este último conjunto, pues a pesar de tener numerosas especies, a ellas corresponde el quinto valor más alto del cociente especies/género (2.88).

\section{Tipos de vegetación}

En la región de Nizanda se reconocieron los siguientes tipos de vegetación: bosque de galería, matorral espinoso, matorral xerófilo, sabana, selva baja caducifolia, selva mediana subcaducifolia y subperennifolia, y vegetación acuática y subacuática. Además, una proporción todavía relativamente pequeña de la superficie corresponde a vegetación secundaria y sistemas agropecuarios. Sólo la selva baja caducifolia (SBC) y las sabanas forman grandes manchones; los tipos de vegetación restantes están restringidos a condiciones microambientales particulares y ocupan extensiones menores.

La variación espacial tanto de la composición florística como de la estructura de la vegetación puede ser abrupta (por ejemplo, las transiciones entre la SBC y la sabana, y entre la SBC y el matorral xerófilo) o gradual (como entre la SBC y los diversos tipos de selvas medianas, o como entre la SBC y el matorral espinoso). Estas diferencias obedecen a las diversas maneras en que cambian en el espacio las características litológicas, fisiográficas, edáficas o incluso los regímenes de disturbio (Fig. 2). En las siguientes secciones se describen de manera general los tipos de vegetación y se ofrecen más detalles sobre la relación entre la vegetación y la heterogeneidad ambiental.

a) Selva baja caducifolia

Además de ser el tipo de vegetación predominante en la región, posiblemente también sea el más heterogéneo, pues presenta notables variaciones fisonómicas y de composición entre los sitios donde se desarrolla. En general, el dosel de la selva baja caducifolia (SBC) se ubica a una altura de alrededor de $7 \mathrm{~m}$, aunque se encuentran algunos árboles emergentes de hasta $15 \mathrm{~m}$, principalmente del género Bursera. La cobertura supera $350 \%$ y en el dosel no hay estratos bien definidos. En esta comunidad son muy comunes las plantas trepadoras, principalmente herbáceas y leñosas delgadas. La diversidad es alta y en general no se reconoce una dominancia clara por parte de alguna especie; de hecho, muchas de ellas pueden ser catalogadas como raras.

La variación más notable de la SBC está claramente asociada con el tipo de material parental sobre el que se desarrolla. Cuando crece sobre esquistos, la SBC está fuertemente dominada por diversas especies de Fabaceae (e.g. Lonchocarpus emarginatus y 


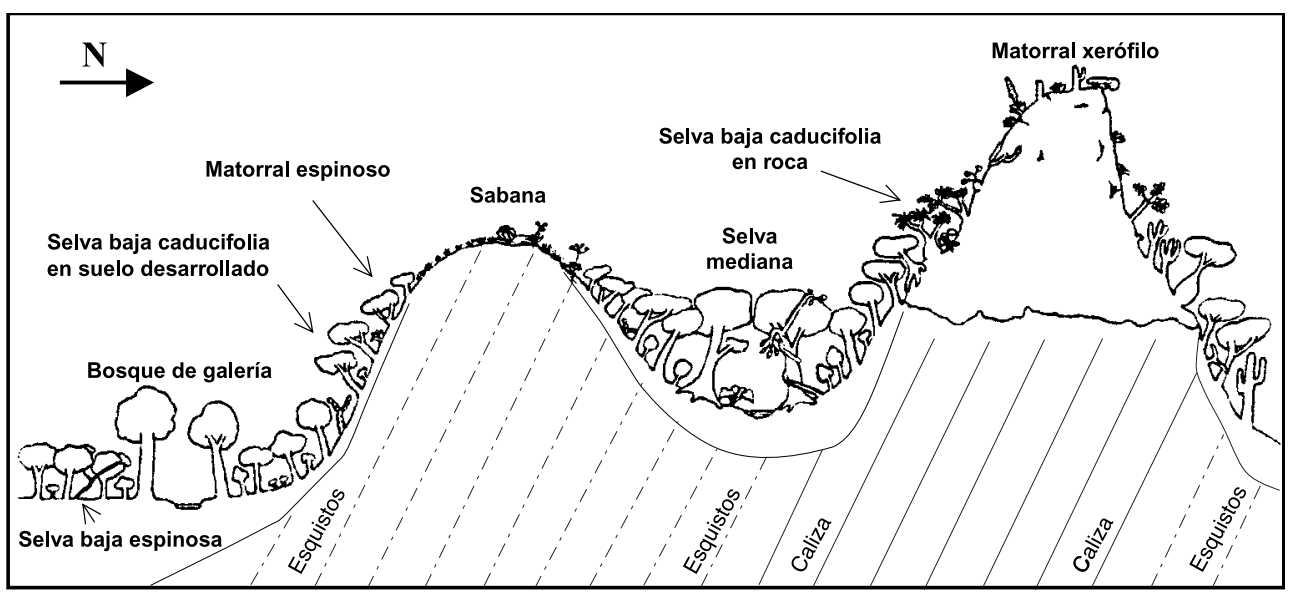

Fig. 2. Diagrama de la vegetación de la región de Nizanda.

Myrospermum frutescens) y Mimosaceae (e.g. Acacia picachensis y Havardia campylacantha), aunque también son frecuentes representantes de otras familias como Coccoloba liebmannii (Polygonaceae), Gyrocarpus mocinnoi (Hernandiaceae), Pachycereus pecten-aboriginum (Cactaceae) y Tabebuia impetiginosa (Bignoniaceae), entre otras. En el sotobosque destacan por su abundancia las Cactaceae Mammillaria voburnensis var. collinsii, Opuntia puberula y Pilosocereus collinsii, las Fabaceae Aeschynomene purpusii y Dalea carthagenensis, así como Haplophyton cimicidum (Apocynaceae) y Jacquinia macrocarpa (Theophrastaceae). En este ambiente las epífitas son en general escasas, pero Clowesia dodsoniana (Orchidaceae) y Tillandsia concolor (Bromeliaceae) llegan a tener una presencia notable. Las trepadoras herbáceas de las familias Asclepiadaceae, Convolvulaceae y Dioscoreaceae son muy frecuentes, y junto con algunas trepadoras leñosas de las familias Bignoniaceae, Malpighiaceae y Sapindaceae confieren a la vegetación una fisonomía intrincada. Agave angustifolia (Agavaceae) es una suculenta muy conspicua en esta comunidad.

Cuando la SBC hace contacto con la sabana, en ocasiones la primera penetra hacia la última a lo largo de cañadas donde se conservan niveles más altos de humedad; en estos sitios destaca Poeppigia procera (Caesalpiniaceae) por su colorido cuando florece, aunque también son frecuentes Godmania aesculifolia (Bignoniaceae), Luehea candida (Tiliaceae) y Swietenia humilis (Meliaceae).

La SBC que se desarrolla sobre roca caliza expuesta (SBCr) es particularmente interesante por su composición florística (Pérez-García et al., 1998a). Esta comunidad incluye especies arbóreas rupícolas como F. pertusa, Ficus petiolaris, y F. ovalis (Moraceae) y arborescentes como Beaucarnea sp. nov. ined. (Nolinaceae), así como plantas capaces de crecer en suelos rocosos poco desarrollados, tales como Bursera excelsa (Burseraceae), Jacaratia mexicana (Caricaceae), Plumeria rubra (Apocynaceae), Pseudobombax ellipticum 
(Bombacaceae), Recchia connaroides (Simaroubaceae) y Guaiacum coulteri (Zygophyllaceae). Entre las especies características de esta comunidad se encuentran Agave nizandensis (Agavaceae), Anthurium nizandense, A. cerrobaulense (Araceae), Cyrtopodium paniculatum, Mesadenus lucayanus (Orchidaceae), Pereskiopsis kellermannii (Cactaceae) y la trepadora Matelea cyclophylla (Asclepiadaceae). En los sitios escarpados donde es frecuente Comocladia engleriana las epífitas son muy abundantes, en particular Tillandsia caputmedusae, T. concolor y T. schiedeana (Bromeliaceae), y Barkeria whartoniana, Cohniella cebolleta, Encyclia sp. nov. ined., E. parviflora y Myrmecophila aff. tibicinis (Orchidaceae). En algunas porciones de esta comunidad existen manchones boscosos prácticamente monoespecíficos de Neobuxbaumia scoparia (Cactaceae) que, si bien no son muy extensos, suelen ser muy densos.

b) Matorral espinoso

El matorral espinoso (ME) tiene una fisonomía semejante a la de la SBC sobre esquistos (Pennington y Sarukhán, 1998). La distinción entre ambas comunidades se basó en que el ME presenta un dosel más bajo $(<4 \mathrm{~m})$, en el que predominan tanto plantas ramificadas desde la base como las que presentan espinas laterales, principalmente de la familia Mimosaceae. EI ME se desarrolla sobre esquistos y por lo general tiene una posición transicional entre las SBC y las sabanas.

En el conjunto de elementos arbóreos característicos del ME se registran: Acacia cochliacantha (Mimosaceae), Amphipterygium adstringens (Julianiaceae), Bursera excelsa (Burseraceae), Castela retusa (Simaroubaceae), Gliricidia sepium (Fabaceae), Hintonia latiflora (Rubiaceae), Mimosa goldmanii (Mimosaceae), Pseudosmodingium multifolium (Anacardiaceae) y Sideroxylon celastrinum (Sapotaceae). Dentro de los arbustos más frecuentes están Cordia oaxacana (Boraginaceae) y Lippia nutans (Verbenaceae); entre las enredaderas destaca la urticante Tragia mexicana (Euphorbiaceae).

En las laderas orientadas hacia el norte, las plantas del ME retienen las hojas por más tiempo, fenómeno que es indicativo de un carácter más mesofítico. Esta condición se refleja también en la composición, ya que las especies espinosas son menos abundantes. Son comunes en estos ambientes Croton niveus (Euphorbiaceae), Mimosa antioquiensis, M. mellii (Mimosaceae) y algunas Asteraceae arbustivas decumbentes como Perymenium aff. grande. Tales diferencias sugieren que además del matorral xerófilo, en la región existen otros dos tipos distintos de matorrales, los espinosos y los subinermes; esta posibilidad tendrá que ser examinada en futuros estudios.

c) Matorral xerófilo

En los escarpes y pedregales de los cerros kársticos se presentan manchones aislados de vegetación xerofítica. En la clasificación de Miranda y Hernández-X. (1963), éstos podrían ser catalogados como matorrales crasi-rosulifolios espinosos, o como cardonales, debido a la abundante presencia de elementos crasicaules afilos. De acuerdo con Rzedowski (1978), ambas categorías corresponden al matorral xerófilo; por tal razón, y considerando que no existen otros matorrales xerofíticos en la región, se decidió utilizar este término. 
Los matorrales xerófilos (MX) están constituidos fundamentalmente por un estrato bajo $(<1 \mathrm{~m})$ dominado por plantas rosetófilas, entre las que destacan la Agavaceae Agave ghiesbreghtii y Bromeliaceae espinosas como Hechtia caudata y $H$. rosea, o inermes, como Tillandsia setacea. Junto a ellas crecen plantas perennes, ejemplificadas por Cnidoscolus aconitifolius (Euphorbiaceae), Echeveria acutifolia (Crassulaceae), Encyclia sp. nov. ined., E. hanburii (Orchidaceae), Milla oaxacana (Alliaceae), Pilea microphylla (Urticaceae), Tillandsia concolor y T. macdougallii (Bromeliaceae), Zephyranthes nelsonii (Amaryllidaceae), así como las cactáceas, tanto globosas (Mammillaria voburnensis var. collinsii y $M$. albilanata), como cilíndricas rastreras (Selenicereus coniflorus). La mayoría de estas especies están casi restringidas a este tipo de vegetación, ya que sólo se comparten con la SBCr.

El estrato arbóreo y el arbustivo son discontinuos, muy bajos, y se distinguen por la presencia de Comocladia engleriana (Anacardiaceae), Ficus ovalis (Moraceae) y Pseudosmodingium multifolium (Anacardiaceae), aunque también son características Bursera schlechtendalii (Burseraceae) y otras tres especies exclusivas de esta comunidad: Cephalocereus nizandensis (Cactaceae), Jatropha oaxacana (Euphorbiaceae) y Pittocaulon velatum var. tzimolense (Asteraceae). En los límites del MX con la SBC son abundantes Philodendron warszewiczii (Araceae), Plumeria rubra (Apocynaceae) y Solandra nizandensis (Solanaceae).

Las comunidades de MX de Nizanda forman conjuntos muy vistosos, notablemente diferentes de los otros tipos de vegetación presentes, que han llamado la atención de los botánicos que han visitado la zona, quienes incluso los han reconocido como verdaderos jardines naturales de roca. En la descripción de Agave nizandensis (Agavaceae), Ladislaus Cutak (1951: 143) reseña que, al conducirlo al lugar, Thomas MacDougall le indicó que en estos sitos: "you will find one of the greatest concentrations of desert plants in this region", y que esto le haría "forget all the inconveniences we have experienced so far and revel in a plantsman's paradise". Los afloramientos rocosos de caliza de características similares son frecuentes en otras porciones del Istmo de Tehuantepec y en ellos también se han encontrado especies endémicas, por ejemplo, Agave guiengola en el cerro Guiengola (Torres-Colín, 1989) y Phragmipedium xerophyticum en la región de los Chimalapas (Soto et al., 1990).

\section{d) Sabana}

La sabana se distribuye en cimas y pendientes de los lomeríos de esquistos, sobre todo en la parte norte de la zona de estudio, en áreas con suelo muy poco desarrollado y posiblemente oligotrófico. El papel del factor edáfico parece estar modificado o incluso intensificado por la ocurrencia ocasional de fuegos periódicos que pueden ser naturales o antropogénicos. La presencia de sabana en la región ha sido citada por varios autores desde la primera mitad del siglo XX (Williams, 1939; Goldman, 1951; Duellman, 1960). Además, fotografías aéreas de la región tomadas en 1961 y 1991 demuestran que en este periodo de 30 años los límites de éstas se mantuvieron invariables y nuestras observaciones de campo indican lo mismo. Tales evidencias, junto con las claras limitaciones edáficas, la presencia de numerosas especies típicas de este tipo de vegetación, así como la información proporcionada por los pobladores de la región, apoyan fuertemente la idea de que la sabana es un tipo de vegetación natural en Nizanda. No obstante, en la actualidad 
existe una tendencia creciente a utilizar algunas porciones de las sabanas para actividades ganaderas, y ello indudablemente está teniendo repercusiones en las características de este tipo de vegetación.

La sabana está dominada por hierbas amacolladas de las familias Poaceae (26 especies), entre las que destacan las pertenecientes a los géneros Andropogon, Aristida, Bouteloua, Cenchrus, Digitaria, Hackelochloa, Heteropogon, Hyparrhenia, Panicum, Paspalum, Schizachyrium, Thrasya, Trachypogon y Urochloa, y Cyperaceae (11 especies), representadas por los géneros Abildgaardia, Bulbostylis, Cyperus y Rhynchospora. Además, son muy comunes las Asteraceae (15 especies) y las Fabaceae (25 especies).

Entre las plantas características de este tipo de vegetación, que no se encuentran en los otros, se tiene a: Alophia drummondii (Iridaceae), Bletia purpurea (Orchidaceae), Byrsonima crassifolia (Malpighiaceae), Calea urticifolia (Asteraceae), Calliandra juzepczukii (Mimosaceae), Chamaecrista diphylla, C. flexuosa, C. serpens (Caesalpiniaceae), Curculigo scorzonerifolia (Hypoxidaceae), Eriosema crinitum (Fabaceae), Krameria revoluta (Krameriaceae), Manfreda pubescens (Agavaceae), Melocactus ruestii (Cactaceae), Pectis saturejoides (Asteraceae), Tephrosia nitens, Zornia megistocarpa, Z. reticulata (Fabaceae), Psidium guineense, $P$. hypoglaucum y $P$. salutare (Myrtaceae).

e) Selva mediana

Este tipo de vegetación se desarrolla en sitios donde la humedad edáfica tiende a ser mayor, por lo cual está básicamente representado por comunidades ribereñas. La fenología foliar de la selva mediana (SM) está fuertemente ligada a la heterogeneidad de la humedad del suelo, lo que permite hacer una distinción entre selvas medianas subperennifolias y subcaducifolias.

La SM subperennifolia se distribuye en las orillas de los arroyos con caudales permanentes. Las especies más comunes de este tipo de vegetación son: Annona squamosa (Annonaceae), Andira inermis (Fabaceae), Augusta rivalis (Rubiaceae), Croton niveus (Euphorbiaceae), Cynometra oaxacana (Caesalpiniaceae), Enterolobium cyclocarpum (Mimosaceae), Ficus insipida (Moraceae), Hemiangium excelsum (Hippocrateaceae), Hiraea reclinata (Malpighiaceae), Inga vera (Mimosaceae), Malmea depressa (Annonaceae), Rondeletia leucophylla (Rubiaceae) y Tabebuia pentaphylla (Bignoniaceae). En el sotobosque es muy común Piper sp. (Piperaceae) y por el tamaño de las hojas destacan los individuos de Xanthosoma robustum (Araceae).

A orillas de los ríos estacionales la vegetación es análoga a una SM subcaducifolia. Entre las especies comunes en estos sitios destacan Calycophyllum candidissimum (Rubiaceae), Coccoloba barbadensis (Polygonaceae), Guazuma ulmifolia (Sterculiaceae), Licania arborea (Chrysobalanaceae), Morisonia americana (Capparaceae) y otras especies típicas de la SBC.

En las cañadas húmedas y en las partes altas del Cerro Naranjo, por encima de los 500 m s.n.m., se presenta otra SM subcaducifolia. Este tipo de vegetación es sin duda el menos explorado. En esta comunidad se presentan algunos elementos típicos de selvas más húmedas como Chamaedorea graminifolia (Arecaceae), Cecropia peltata (Cecropiaceae), Enterolobium cyclocarpum (Mimosaceae), Brosimum alicastrum (Moraceae), Piper auritum (Piperaceae), Rheedia edulis (Clusiaceae), Triphora gentianoides y T. aff. trianthophora 
(Orchidaceae). En el cauce rocoso de los arroyos son frecuentes los helechos Adiantum princeps, A. tetraphyllum, Pityrogramma calomelanos (Pteridaceae) y Tectaria heracleifolia (Tectariaceae). Cabe mencionar que este tipo de vegetación, junto con la SBC que lo rodea, es el que presenta el mejor estado de conservación en la región. Reflejo de ello es la presencia de fauna que ya ha desaparecido de muchas otras partes de las regiones tropicales del país, entre la que destacan el mono araña (Ateles geoffroyi subsp. vellerosus), siendo éste en la actualidad su registro más septentrional en la vertiente del Pacífico.

\section{f) Bosque de galería}

En la planicie de inundación del Río Verde, donde el caudal superficial se infiltra hasta desaparecer por completo, existía un bosque de galería dominado por Astianthus viminalis (Bignoniaceae). Desafortunadamente, esta comunidad ha desaparecido casi por completo debido a las actividades agrícolas, por lo que sólo permanecen algunos fragmentos aislados a lo largo del cauce del río. Tal situación dificulta estimar la extensión original de la comunidad y reconstruir con exactitud su composición florística, aunque todavía conviven con dicha especie dominante otras entre las que destacan Thouinidium decandrum (Sapindaceae), Lantana hirta y Vitex mollis (Verbenaceae). Los fragmentos remanentes de este tipo de vegetación permiten suponer que se trataba de una comunidad continua pero estrecha, restringida al borde del río y cuyo dosel más o menos abierto alcanzaba una altura de $15 \mathrm{~m}$.

Los pobladores locales mencionan también la existencia pasada de un matorral ribereño de Baccharis salicifolia (Asteraceae). En el presente, esta comunidad se encuentra reducida a unos cuantos individuos en la desembocadura del arroyo Mazahua. Por otro lado, cerca del poblado La Mata se localiza un bosque de galería de Parkinsonia aculeata (Caesalpiniaceae); los sitios donde éste se encuentra sufren un disturbio constante por pastoreo y por ello es probable que se trate de una comunidad secundaria.

\section{g) Vegetación acuática y subacuática}

Las especies acuáticas y subacuáticas se restringen a cuerpos permanentes y semipermanentes de agua. Sin conformar propiamente un tipo de vegetación, se incluye aquí al conjunto de plantas que comparten afinidad por el agua. Aunque se excluyó de esta categoría a la selva mediana ribereña, en realidad en ocasiones es difícil separar con precisión ambas comunidades. Ejemplo de ello lo constituye un pequeño humedal dominado por el helecho Acrostichum danaeifolium (Pteridaceae), el cual está rodeado por árboles propios de selva mediana. Quizá la vegetación acuática más característica sea la que se localiza en sitios desprovistos de cubierta arbórea, como la comunidad de Ceratopteris thalictroides (Pteridaceae), planta sumergida que se establece en el fondo de los arroyos y cuyas frondas reproductivas son emergentes. También asociadas a sitios de inundación están Fimbristylis spadicea (Cyperaceae), Samolus ebracteatus (Primulaceae) y Typha domingensis (Typhaceae), aunque es probable que su presencia tenga un origen secundario. 
h) Vegetación secundaria

La vegetación de sitios perturbados por las actividades humanas y los estadios sucesionales subsecuentes fueron agrupados bajo el rubro de vegetación secundaria. A pesar de ser un conjunto heterogéneo, en general en ella dominan plantas arvenses, particularmente de las familias Asteraceae, Malvaceae y Poaceae. En el caso de esta última, es notable que los pastos introducidos sean más frecuentes en la vegetación secundaria, mientras que los nativos están prácticamente ausentes, situación inversa a lo que ocurre en las sabanas. Los sitios sucesionales avanzados están frecuentemente dominados por Mimosaceae espinosas, especialmente de los géneros Mimosa y Acacia.

La composición y la dinámica de estas comunidades secundarias son prácticamente desconocidas, a pesar de que su presencia en la región aumenta conforme avanza la degradación de los ecosistemas nativos. Por ello, su estudio, sobre todo con fines de restauración, se hace cada vez más necesario.

\section{DISCUSIÓN}

Las plantas recolectadas durante cinco años en la región de Nizanda permiten apreciar su gran riqueza florística y hacen evidente el papel de la heterogeneidad ambiental y de la vegetación en el mantenimiento de un contingente tan numeroso de especies. Sin considerar a los ejemplares que no han podido ser determinados, el listado aquí elaborado representa un incremento aproximado de $17 \%$ sobre el número de especies registradas en el trabajo de Torres-Colín et al. (1997); esta cifra corrobora la contribución de Nizanda a la conformación de la flora regional del Istmo de Tehuantepec.

La prospección florística ha permitido registrar nuevos taxa para la región. Adiciones notables a la flora istmeña son la familia Buxaceae (con Buxus bartlettii), cuya presencia no era probable en la región por tratarse de un grupo típico de ambientes más húmedos (R. Torres-Colín, com. pers.), Phaseolus oligospermus (Fabaceae), especie previamente conocida desde Chiapas hasta Costa Rica (A. Delgado, com. pers.), y Aristolochia anguicida, cuya distribución conocida se restringía a Centro y Sudamérica, por lo que representa un nuevo registro para el país (B. González, com. pers.). Además, algunos ejemplares recolectados parecen representar nuevas especies para la ciencia.

A pesar de la gran heterogeneidad de la cubierta vegetal de Nizanda, llama la atención que la selva baja caducifolia espinosa no sea conspicua en la región de estudio, ya que Rzedowski (1978) y Pennington y Sarukhán (1998) la citan como la vegetación predominante en la región istmeña. La presencia de individuos dispersos de Pereskia lychnidiflora (Cactaceae) sugiere que hubo comunidades de este tipo en las partes bajas de la cuenca del Río Verde, donde la vegetación original ha sido reemplazada casi completamente por campos agrícolas. Por esta razón, la selva baja caducifolia espinosa fue excluida en este trabajo, a pesar de que todavía persisten extensiones considerables de ella en la Planicie Costera del Istmo.

La heterogeneidad de la cubierta vegetal de Nizanda es un factor clave en la determinación de la riqueza de su flora. Los resultados de este estudio no permiten resolver las numerosas incógnitas acerca de las causas de los patrones observados en la distribución 
de la vegetación, pero sí formular algunas hipótesis. Por ejemplo, el predominio de las Poaceae y Cyperaceae en las sabanas podría estar relacionado con la resistencia de muchas especies de estos grupos al fuego y a las limitaciones hídricas y nutricionales, factores que parecen operar fuertemente en las cimas de los lomeríos de esquistos. De manera análoga, la abundancia de plantas clonales en el matorral xerófilo podría indicar que existen limitaciones para el establecimiento a partir de semillas, y la presencia de elementos crasos perennifolios podría estar correlacionada con una baja fertilidad y con la ausencia de fuegos recurrentes en este tipo de vegetación.

Las plantas epífitas muestran patrones que se ajustan a predicciones teóricas, como el incremento de su abundancia y diversidad hacia elevaciones mayores (Benzing, 1990), en particular en el Cerro Verde ( \pm 500 m s.n.m.) y otros cerros kársticos, sobre todo en sus laderas orientadas al norte. Sin embargo, estas plantas también presentan patrones que carecen de una explicación aparente. Por ejemplo, los sitios con mayor diversidad de epífitas son los escarpes calizos, incluyendo a los matorrales xerófilos y a la SBCr. Este fenómeno puede estar relacionado de forma compleja con factores tan variados como la incidencia del viento, el balance de radiación, el tipo de sustrato y la abundancia de árboles hospederos, entre los que Comocladia engleriana (Anacardiaceae) juega un papel importante.

Las Cactaceae constituyen otro grupo de plantas con patrones complejos de distribución en Nizanda. Sólo unas cuantas especies de esta familia están ampliamente distribuidas en la región, como es el caso de Nopalea karwinskiana, Pachycereus pectenaboriginum y Pilosocereus collinsii. Sin embargo, es más común que las especies muestren preferencias por algún ambiente particular. Por ejemplo, tanto en la SBCr como en el MX de los cerros kársticos, donde los cactos son muy abundantes, se reconocieron muchas especies exclusivas de esos ambientes: Cephalocereus nizandensis, Mammillaria albilanata, Neobuxbaumia scoparia, Pereskiopsis kellermannii y Selenicereus coniflorus. Otras cactáceas que están completamente ausentes de la vegetación de terrenos kársticos también se restringen a ambientes específicos. Tal es el caso de Melocactus ruestii, típicamente presente en las sabanas, y de Pereskia lychnidiflora, que como se mencionó, sus pocos individuos se restringen a lo que parecen ser remanentes de selva baja caducifolia espinosa.

Una peculiaridad notable de la flora de Nizanda es la presencia de elementos propios de ambientes más frescos, por ejemplo Cattleya aurantiaca (Orchidaceae), Echeveria acutifolia (Crassulaceae), Encyclia hanburii (Orchidaceae), Jatropha oaxacana (Euphorbiaceae) y Tillandsia setacea (Bromeliaceae). Aunque esta situación puede representar una respuesta actual al efecto refrescante del viento, su explicación también podría tener un componente histórico.

Un aspecto de la flora de Nizanda que plantea muchas interrogantes es el relativo a sus nexos fitogeográficos. La presencia de especies indicativas de relaciones con la porción centro-occidental de México (Valle de Tehuacán-Cuicatlán, Cuenca del Río Balsas y la vertiente del Pacífico), con el Golfo de México, con el sureste del país, y con América Central, hacen que este asunto amerite a futuro un análisis cuidadoso, y aquí sólo se ofrece una interpretación preliminar.

Rzedowski (1978) ubicó al Istmo de Tehuantepec dentro de la Provincia Florística de la Costa del Pacífico, en la Región Caribea. A una escala más pequeña, García-Mendoza 
y Torres-Colín (1999) consideran que la vertiente pacífica del Istmo de Tehuantepec forma una región propia y distinta del resto de la Planicie Costera del Pacífico. De acuerdo con esta consideración, se esperaría que Nizanda presentara una mayor afinidad fitogeográfica con las otras regiones istmeñas, y que existieran relaciones subsecuentemente decrecientes con la región Costera de Oaxaca (García-Mendoza y Torres-Colín, 1999), y con lo que Lott y Atkinson (en prensa) definen como la región Centro del Pacífico Mexicano. De acuerdo con la concepción de estos últimos autores, Nizanda pertenecería a un conjunto de entidades fitogeográficas anidadas en orden ascendente que conforman una gran región biogeográfica a lo largo de las costas mexicanas del Océano Pacífico, caracterizada por una gran diversidad florística y un alto nivel de endemismo (Gentry, 1995). No obstante, esta conceptualización biogeográfica no es congruente con la presencia en Nizanda de varias especies propias de la vertiente atlántica, como Acacia pringlei (Mimosaceae), Chamaecrista diphylla (Caesalpiniaceae), Euphorbia pteroneura (Euphorbiaceae), Neobuxbaumia scoparia (Cactaceae) y Pleurothallis digitale (Orchidaceae).

La presencia de plantas xerófilas en la vegetación de los sitios kársticos de Nizanda (SBCr y MX), particularmente de Echeveria acutifolia (Crassulaceae), Fouquieria formosa (Fouquieriaceae) y Jatropha oaxacana (Euphorbiaceae), parece estar relacionada con la existencia hipotética en el pasado de una gran zona semiárida que habría incluido a la cuenca del Balsas y el Valle de Tehuacán-Cuicatlán (González-Medrano y Chiang, 1988), y que probablemente estuvo conectada con la región de Nizanda a través de la cuenca del Río Tehuantepec (Acosta-Castellanos, 1992). Si bien en principio se podría suponer que la ruta de colonización de estas especies fue desde el centro hacia el sur del país, GonzálezMedrano (1996) sugirió que grupos de plantas crasas y rosetófilas de origen neotropical que toleran la aridez y que habitan en paisajes kársticos, como Agave, Echeveria, Hechtia y Yucca (todas ellas presentes en el karst de Nizanda), podrían haber colonizado el Desierto Chihuahuense. Esta hipótesis es congruente con la existencia de plantas muy antiguas en ambientes kársticos en el Istmo, por ejemplo Phragmipedium xerophyticum (Soto et al., 1990) y sustenta el planteamiento alternativo de que en los terrenos kársticos del Istmo de Tehuantepec permanecen comunidades relictuales como un vestigio del tránsito de la vegetación xerófila en sentido sur a norte.

El Istmo de Tehuantepec ha sido señalado como una región con un alto nivel de endemismo (Lorence y García-Mendoza, 1989). La evaluación del endemismo de una región donde convergen plantas con distintos orígenes biogeográficos requiere de inventarios de diversas áreas y mapas de distribución detallados de las especies. La escasez de este tipo de información dificulta estimar la magnitud de la fracción endémica de la flora de Nizanda. A pesar de ello, hay claros indicios de que el grado de endemismo de estas plantas es muy variable: Barkeria whartoniana (Orchidaceae) se puede considerar como una especie microendémica, Agave nizandensis (Agavaceae) solamente crece en los cerros calizos de la vertiente pacífica del Istmo, y Pleurothallis digitale (Orchidaceae) también presenta una distribución restringida pero centrada en la vertiente del Golfo de México. Asimismo, Zornia megistocarpa (Fabaceae) sólo se conocía de la localidad tipo en Salina Cruz, mientras que las áreas de distribución de Lonchocarpus sp. nov. ined. 2 y $L$. emarginatus (Fabaceae) están limitadas a las tierras bajas del Istmo de Tehuantepec y la porción aledaña de la costa del Pacífico. Un detalle adicional interesante respecto a las especies endémicas en Nizanda es que éstas se distribuyen diferencialmente entre los 
varios tipos de vegetación. Por ejemplo, en las comunidades ribereñas prácticamente no hay taxa endémicos, mientras que en la vegetación xerofítica sobre los cerros de caliza (SBCr y MX) tal componente es muy conspicuo.

La vegetación de Nizanda manifiesta el papel que ha jugado simultáneamente el Istmo de Tehuantepec a través de la historia de la flora mexicana como punto de contacto y como barrera entre biotas distintas, cuya convivencia enriquece la diversidad de linajes evolutivos presentes en la región. Esto se refleja en la existencia en Nizanda de 119 familias de plantas vasculares registradas hasta ahora. Esta cifra debe tomarse con cautela debido a la heterogeneidad en la circunscripción de las familias en los distintos trabajos y porque las áreas estudiadas son de diferente tamaño; sin embargo, es indudable que la riqueza de familias en Nizanda es una de las más altas en relación con otras áreas tropicales estacionales en México, Por ejemplo, en el Cañón del Zopilote se registraron 79 familias (Gual, 1995); en la Laguna de Mitla, 99 (Lozada, 1994); 103 en la Depresión Central de Chiapas (Reyes-García y Sousa, 1997); 107 en la Estación de Biología Chamela (Lott, 1985); y 124 para toda la región de la Bahía de Chamela (Lott, 1993).

La cubierta vegetal de Nizanda está conformada por un complejo mosaico de diferentes comunidades, asociadas a diversas condiciones ambientales y de disturbio. La realización de análisis cuantitativos más formales de estas relaciones requiere que los esfuerzos de colecta logren un mejor balance entre los diferentes tipos de vegetación; en tal sentido, este estudio puede servir de base para futuras investigaciones que permitan comprender mejor la gran riqueza y complejidad de la flora de la región istmeña.

\section{AGRADECIMIENTOS}

Como en todo trabajo de prospección florística en regiones de gran diversidad, la colaboración de los especialistas es fundamental. En este sentido, agradecemos muy sinceramente a las siguientes personas: Gloria Andrade (Mimosaceae), Daniel F. Austin (Convolvulaceae), Ma. Goreti Campos (Boraginaceae), Antonio Carrillo (Poaceae), Javier Castrejón (Cucurbitaceae), Fernando Chiang (Rutaceae), Alfonso Delgado (Fabaceae), Nelly Diego (Cyperaceae y Apocynaceae), Adolfo Espejo (Commelinaceae), Gabriel Flores (Caesalpiniaceae), Rosa Ma. Fonseca (Anacardiaceae, Hippocrateaceae y Salicaceae), Susana Gama (Stylosanthes), Abisaí García (Liliopsida), Ma. Teresa Germán (Meliaceae), Rosa E. González (Acanthaceae), Rosaura Grether (Mimosaceae), Guillermo Ibarra (Moraceae), Jaime Jiménez (Euphorbiaceae), Rolando Jiménez (Orchidaceae), Rafael Lira (Cucurbitaceae), Lilián López (Crassulaceae), Liliana I. López (varias), Francisco Lorea (Lauraceae), Antonio Lot (Lemnaceae), Lucio Lozada (Asclepiadaceae y otras), Martha Martínez (Euphorbiaceae), Esteban Martínez (Bignoniaceae), Isidro Méndez (Scrophulariaceae), Michael Nee (Solanaceae), Rodolfo Noriega (Rubiaceae), Helga Ochoterena (Rubiaceae), Darisol Pacheco (Poaceae), Hermilo Quero (Arecaceae), Ivón M. Ramírez (Bromeliaceae), Francisco Ramos (Buxaceae), Clara H. Ramos (Asteraceae), Antonio Reyes (Nyctaginaceae), Jerónimo Reyes (Cactaceae), Jon Ricketson (Myrsinaceae), Lourdes Rico (Mimosaceae), Gerardo Salazar (Orchidaceae), Ivonne Sánchez (Amaranthaceae), Jorge Sánchez (Poaceae), Miguel A. Soto (Orchidaceae), Mario Sousa (leguminosas), Oswaldo Téllez (Dioscoreaceae y leguminosas), Rafael Torres (legumino- 
sas), Leticia Torres (Macroptilium), Ernesto Velázquez (Pteridophyta), José Luis Villaseñor (Asteraceae) y Tom Wendt (Polygalaceae).

Mario Sousa y el personal del Herbario Nacional (MEXU) otorgaron numerosas facilidades para el desarrollo del trabajo. Fernando Chiang, Emily Lott, Armando Luis, Mario Sousa, Rafael Torres, José Luis Villaseñor y dos revisores anónimos hicieron valiosas observaciones a versiones preliminares de este manuscrito. Oswaldo Núñez, Edwin Lebrija, Liliana López, Adriana Osnaya y Balam Pérez participaron en el trabajo de campo. La colaboración de Marco Antonio Romero fue fundamental para el manejo de la base de datos. Los habitantes de Nizanda brindaron en todo momento su apoyo para la realización del trabajo de campo; un agradecimiento muy especial merece la familia Reyes Manuel. Este estudio fue posible gracias al apoyo financiero otorgado por la CONABIO (proyectos G018 y L085). El primer autor agradece al Fondo Mexicano para la Conservación de la Naturaleza por la beca otorgada.

\section{LITERATURA CITADA}

Anónimo. 1981a. Atlas nacional del medio físico. Secretaría de Programación y Presupuesto. México, D.F. 223 pp.

Anónimo. 1981b. Juchitán E15-10, D15-1, escala 1:250,000. Carta topográfica. Instituto Nacional de Estadística, Geografía e Informática. México, D.F.

Anónimo. 1984a. Carta de efectos climáticos regionales mayo-octubre. Juchitán E15-10 D15-1, escala 1:250,000. Secretaría de Programación y Presupuesto, México, D.F.

Anónimo. 1984b. Carta de efectos climáticos regionales noviembre-abril. Juchitán E15-10 D15-1, escala 1:250,000. Secretaría de Programación y Presupuesto, México, D.F.

Anónimo. 1988a. Ciudad Ixtepec E15C63, escala 1:50,000. Carta topográfica. Instituto Nacional de Estadística, Geografía e Informática. Aguascalientes.

Anónimo. 1988b. Santo Domingo E15C64, escala 1:50,000. Carta topográfica. Instituto Nacional de Estadística, Geografía e Informática. Aguascalientes.

Acosta-Castellanos, S. 1992. La parte alta de la cuenca del Río Tehuantepec, Oaxaca: flora, comunidades y relaciones fitogeográficas. Tesis (Maestría en Ciencias), Escuela Nacional de Ciencias Biológicas, Instituto Politécnico Nacional. México, D.F. 82 pp.

Benzing, D. H. 1990. Vascular epiphytes. General biology and related biota. Cambridge University Press. Nueva York. 354 pp.

Bravo, H. y T. MacDougall. 1958. Revisión del género Neodawsonia. Anales Inst. Biol. Univ. Nac. Autón. México, Ser. Bot. 29: 73-87.

Brummitt, R. K. y C. E. Powell (eds.). 1992. Authors of plant names. The Royal Botanic Gardens. Kew. $732 \mathrm{pp}$.

Chelton, D. B., M. H. Freilich y S. K. Esbensen. Sin fecha. Satellite observations of the wind jets off Central America. Oregon State University. (www.oce.orst.edu/po/research/windjets/index.html)

Cronquist, A. 1981. An integrated system of classification of flowering plants. Columbia University Press. Nueva York. 1262 pp.

Cutak, L. 1951. A new dwarf Mexican Agave. Cact. Succ. J. (US) 23: 143-145.

Dahlgren, R. M. T., H. T. Clifford y P. F. Yeo. 1985. The families of the Monocotyledons. Structure, evolution, and taxonomy. Springer-Verlag. Berlín. 520 pp.

Duellman, W. E. 1960. A distributional study of the amphibians of the Isthmus of Tehuantepec, Mexico. Univ. Kansas Pub. 13: 19-72.

García, E. 1988. Modificaciones al sistema de clasificación climática de Köppen. 4a ed. Edición de la autora. México, D.F. 219 pp. 
García-Mendoza, A. y R. Torres-Colín. 1999. Estado actual del conocimiento sobre la flora de Oaxaca, México. In: Vásquez Dávila, M. A. (ed.). Sociedad y naturaleza en Oaxaca 3. Instituto Tecnológico Agropecuario de Oaxaca. Oaxaca. pp. 50-86.

Gentry, A. H. 1995. Diversity and floristic composition of neotropical dry forests. In: Bullock, S. H., H. A. Mooney y E. Medina (eds.). Seasonally dry tropical forests. Cambridge University Press, Cambridge. pp.146-194.

Goldman, E. A. 1951. Biological investigations in Mexico. Smithsonian Misc. Collect. 115: 1-476.

González-Medrano, F. 1996. Algunos aspectos de la evolución de la vegetación de México. Bol. Soc. Bot. México 58: 129-136.

González-Medrano, F. y F. Chiang. 1988. Diversidad florística y fitogeografía de las zonas áridas del centro y del sur de México. Simposio sobre diversidad biológica de México. Instituto de Biología, Universidad Nacional Autónoma de México. México, D.F. pp. 20-21.

Gual, M. 1995. Cañón del Zopilote (Área Ventana Vieja). In: Diego-Pérez, N. y R. Fonseca (eds.). Estudios florísticos de Guerrero No. 6. Facultad de Ciencias, Universidad Nacional Autónoma de México. México, D.F. 39 pp.

Jáuregui, E. 1984. El clima de Salina Cruz, Oax. En: El puerto industrial de Salina Cruz, Oaxaca. Seminario Franco-Mexicano, julio 1982. Instituto de Geografía, UNAM y Centro de Investigaciones y Documentación de América Latina (CREDAL/CNRS). México, D.F. 187 pp.

Lorence, D. H. y A. García-Mendoza, 1989. Oaxaca, Mexico. In: Campbell, D. G. y H. D. Hammond (eds.). Floristic inventory of tropical countries: the status of plant systematics, collections, and vegetation, plus recommendations for the future. New York Botanical Garden. Nueva York. pp. 253-269.

Lott, E. J. 1985. Listados florísticos de México III. La Estación de Biología de Chamela, Jalisco. Instituto de Biología, Universidad Nacional Autónoma de México. México, D.F. 47 pp.

Lott, E. J. 1993. Annotated checklist of the vacular flora of the Chamela Bay region, Jalisco, Mexico. Occas. Pap. Calif. Acad. Sci. 148: 1-60.

Lott, E. J. y T. H. Atkinson. En prensa. Biodiversidad y fitogeografía de Chamela-Cuixmala, Jalisco. In: Noguera F. A., J. Vega y M. Quesada (eds.). Historia natural de Chamela. Instituto de Biología, Universidad Nacional Autónoma de México. México, D.F.

Lozada, L. 1994. Laguna de Mitla. In: Diego-Pérez, N. y R. Fonseca (eds.). Estudios florísticos de Guerrero No. 2. Facultad de Ciencias, Universidad Nacional Autónoma de México. México, D.F. $44 \mathrm{pp}$.

Matuda, E. 1959. Nuevas especies Anthurium de Oaxaca. Bol. Soc. Bot. México 24: 35-38.

Miranda, F. y E. Hernández-X. 1963. Los tipos de vegetación en México y su clasificación. Bol. Soc. Bot. México 28: 29-179.

Moran, R. C. y R. Riba (eds.). 1995. Volumen I. Psilotaceae a Salviniaceae. In: Davidse, G., M. Sousa y S. Knapp (eds.). Flora Mesoamericana. Universidad Nacional Autónoma de México, Missouri Botanical Garden y The Natural History Museum (London). México, D.F. 470 pp.

Pennington, T. D. y J. Sarukhán. 1998. Manual para la identificación de los principales árboles tropicales de México. Universidad Nacional Autónoma de México y Fondo de Cultura Económica, México, D.F. 517 pp.

Pérez-García, E. A., C. Gallardo y J. Meave. 1998a. Floristic differentiation within and between xerophytic habitat islands in a seasonally dry forest region. Abstracts of the 1998 Annual Meeting of the Association for Tropical Biology and 49th Annual Meeting of the American Institute of Biological Sciences, Baltimore, Maryland, agosto 2-6, 1998.

Pérez-García, E. A, C. Gallardo y J. Meave. 1998b. Heterogeneidad vegetacional en un paisaje complejo del trópico estacionalmente seco en el Istmo de Tehuantepec, Oaxaca. Libro de Resúmenes, VII Congreso Latinoamericano de Botánica y XIV Congreso Mexicano de Botánica, 18 al 24 de octubre de 1998. Sociedad Botánica de México. México, D.F. pp. 235.

Peterson, A. T., J. Soberón y V. Sánchez-Cordero. 1999. Conservatism of ecological niches in evolutionary time. Science 285: 1265-1267. 
Reyes-García, A. y M. Sousa. 1997. Listados florísticos de México XVII. Depresión Central de Chiapas. La selva baja caducifolia. Instituto de Biología, Universidad Nacional Autónoma de México. México, D.F. 41 pp.

Rodrigo Álvarez, L. 1994. Geografía general del estado de Oaxaca. Carteles Editores. Oaxaca. 456 pp.

Rzedowski, J. 1978. Vegetación de México. Editorial Limusa. México, D.F. 432 pp.

Soto, M. A., G. Salazar y E. Hágsater. 1990. Phragmipedium xerophyticum, una nueva especie del sureste de México. Orquídea (Méx.) 12: 1-10

Soto-Arenas, M. A. 1993. Barkeria melanocaulon y Barkeria whartoniana. Orquídea (Méx.) 13: 233-244.

Torres-Colín, L. M. 1989. Estudio florístico y descripción de la vegetación del cerro Guiengola, en el Istmo de Tehuantepec, Oaxaca. Tesis de licenciatura, Escuela Nacional de Estudios Profesionales Iztacala, Universidad Nacional Autónoma de México. Iztacala. 81 pp.

Torres-Colín, R., L. Torres-Colín, P. Dávila-Aranda y J. L. Villaseñor-Ríos. 1997. Listados florísticos de México XVI. Flora del Distrito de Tehuantepec, Oaxaca. Instituto de Biología, Universidad Nacional Autónoma de México. México, D.F. 68 pp.

Wendt, T. 1998. Composición, afinidades florísticas y orígenes de la flora arbórea del dosel de los bosques tropicales húmedos de la vertiente mexicana del Atlántico. In: Ramamoorthy, T. P., R. Bye, A. Lot y J. Fa (comps.). Diversidad biológica de México. Orígenes y distribución. Universidad Nacional Autónoma de México. México, D.F. pp. 581-664.

Williams, L. 1939. Árboles y arbustos del Istmo de Tehuantepec, México. Lilloa 4: 137-171.

Zizumbo, D. y P. Colunga. 1980. La utilización de los recursos naturales entre los huaves de San Mateo del Mar, Oaxaca. Tesis de Licenciatura, Facultad de Ciencias, Universidad Nacional Autónoma de México. México, D.F. 375 pp. 


\section{APÉNDICE}

Lista florística de la región de Nizanda, Oaxaca. Se indica el tipo de vegetación donde se recolectaron los ejemplares. $\mathrm{SAB}=$ sabana, $\mathrm{SBC}=$ selva baja caducifolia, $\mathrm{ME}=$ matorral espinoso, $\mathrm{MX}=$ matorral xerófilo, $\mathrm{SM}=$ selva mediana, $\mathrm{BG}=$ bosque de galería, $\mathrm{VA}=$ vegetación acuática, VS = vegetación secundaria, ${ }^{*}=$ no registrado en Torres et al. (1997), $\left({ }^{*}\right)=$ ausente en dicha obra pero encontrado recientemente en el Distrito de Tehuantepec (R. Torres-Colín, com. pers.), CGH = Claudia Gallardo Hernández, ELT = Edwin Lebrija Trejos, LLO = Liliana López Olmedo, JM = Jorge Meave, AOP = Adriana Osnaya Palomares, $\mathrm{EAPG}=$ Eduardo A. Pérez-García.

Familias, Especies y Colectas

Tipo de vegetación

SAB SBC ME MX SM BG VA VS

\section{PTERIDOPHYTA}

\section{ASPLENIACEAE}

* Asplenium hoffmannii Hieron. CGH 1915, EAPG 2010

$X$

LOMARIOPSIDACEAE

* Bolbitis portoricensis (Spreng.) Hennipman

$\mathrm{X}$ EAPG 2008

POLYPODIACEAE

* Microgramma nitida (J. Sm.) A. R. Sm. $X$ EAPG 1262

Niphidium crassifolium (L.) Lellinger $X$ EAPG 2009

PTERIDACEAE

* Acrostichum danaeifolium Langsd. et Fisch.

EAPG 1089

*Adiantum deflectens Mart. X CGH 1900

* Adiantum lunulatum Burm. f. $\quad \mathrm{X} \quad \mathrm{X}$

ELT 38, JM 2312

Adiantum princeps T.Moore CGH 1923, JM 2107, EAPG 1492

$\mathrm{X}$

* Adiantum tetraphyllum Humb. et Bonpl. ex Willd.

CGH 1916 
Acta Botanica Mexicana (2001), 56: 19-88

Apéndice. Continuación.

Familias, Especies y Colectas

Tipo de vegetación

SAB SBC ME MX SM BG VA VS

* Adiantum villosum L.

$X$ EAPG 1493

* Ceratopteris thalictroides (L.) Brongn.

$X$ CGH 1965, EAPG 1033

* Cheilanthes alabamensis (Buckley) Kunze CGH 1834, EAPG 1147

* Cheilanthes lozanii (Maxon) R. M. Tryon et A. F. Tryon ELT 33, EAPG 1788

* Cheiloplecton rigidum (Sw.) Fée var. rigidum

LLO 64, JM 2347

* Hemionitis pinnatifida Baker ELT 31

* Megalastrum pulverulentum (Poir.) A. R. Smith et R. C. Moran EAPG 1417

Pityrogramma calomelanos (L.) Link $\quad \mathrm{X}$ EAPG 1416

* Pityrogramma dealbata (C. Presl) R. M. Tryon

EAPG 200

Tectaria heracleifolia (Willd.) Underw.

EAPG 1471, 2014

THELYPTERIDACEAE

* Macrothelypteris torresiana (Gaudich.)

Ching

EAPG 1215, 2001

* Thelypteris hispidula (Decne.) C. F. Reed X

EAPG 1487, 2012

SCHIZAEACEAE

*Anemia aff. familiaris Mickel X EAPG 1956

*Anemia hirsuta (L.) Sw. X

EAPG 2043

* Lygodium venustum Sw. X

EAPG 1552 
Apéndice. Continuación.

Familias, Especies y Colectas

Tipo de vegetación

SAB SBC ME MX SM BG VA VS

\section{CYCADOPHYTA}

ZAMIACEAE

* Zamia loddigesii Miq. var. spartea (A.

DC.) Schuster

$X$

JMC 2388

\section{MAGNOLIOPHYTA}

\section{MAGNOLIOPSIDA}

\section{ACANTHACEAE}

Aphelandra scabra (Vahl) Sm.

CGH 2329, JM 1869

* Aphelandra schiedeana Schltdl. et Cham. X EAPG 1428

Barleria oenotheroides Dum. Cours. X EAPG 1049, 1413

Bravaisia integerrima (Spreng.) Standl. X JM 1938, EAPG 1099

Holographis leticiana T. F. Daniel X EAPG 1440

Justicia caudata A.Gray

CGH 2285, 2335

Ruellia inundata Kunth

CGH 2324

Ruellia pringlei Fernald

EAPG 1139, 1352

Tetramerium obovatum T. F. Daniel X EAPG 1461

\section{ACHATOCARPACEAE}

Achatocarpus nigricans Triana JM 1806

\section{AIZOACEAE}

* Trianthema portulacastrum L. JM 2337 
Acta Botanica Mexicana (2001), 56: 19-88

Apéndice. Continuación.

Familias, Especies y Colectas

Tipo de vegetación

SAB SBC ME MX SM BG VA VS

AMARANTHACEAE

Alternanthera mexicana Moq. CGH 1649

Alternanthera pycnantha (Benth.) Standl. CGH 1698, EAPG 922, 1363

Alternanthera aff. pycnantha (Benth.)

Standl.

CGH 1631

* Amaranthus scariosus Benth. CGH 1971

* Chamissoa acuminata Mart. var. swansonii Sohmer EAPG 735, 902

* Gomphrena globosa L. EAPG 1534, 1535

Iresine calea (Ibáñez) Standl. EAPG 1074

$\begin{array}{ll} & x \\ x & x \\ x & \\ x & x\end{array}$

* Iresine aff. diffusa Humb. et Bonpl. ex Willd.

CGH 1685

* Iresine aff. interrupta Benth. EAPG 995

* Iresine sp. EAPG 900, 934

Lagrezia monosperma (Rose) Standl. CGH 1637, 1651, 1700, 1689, EAPG 746, 820, 890,

ANACARDIACEAE

Comocladia engleriana Loes.

EAPG 951, 1028, 1056

Pseudosmodingium multifolium Rose

EAPG 719, 953

* Spondias purpurea L. CGH 1601, 1985, EAPG 1054, 1678

* Spondias radlkoferi Donn. Sm.

X $\quad X$

CGH 1607, EAPG 1098, 1496

$X$

X $\quad X \quad X$

$X$

$X$

$x$

X

$X$

$x$

$x$

X 
Apéndice. Continuación.

Familias, Especies y Colectas

Tipo de vegetación

SAB SBC ME MX SM BG VA VS

ANNONACEAE

* Annona reticulata $\mathrm{L}$. CGH 1626

Annona squamosa L. CGH 1452, 1498, JM 1817, 1877, EAPG 1156

(*) Malmea depressa (Baill.) R. E. Fr. CGH 1450, 1625, JM 1812, 2283, EAPG 732, 867, 942, 1157, 1476, 1702

Sapranthus microcarpus (Donn. Sm.) R. E. Fr.

CGH 1669, JM 1820, EAPG 862, 905,

$x$

$X$ 1150

APOCYNACEAE

Aspidosperma megalocarpon Müll. Arg. CGH 1558

Haplophyton cimicidum A. DC. CGH 1678, 1847, 2262, 2323, JM 1783, 2292, EAPG 1236

* Mesechites trifida (Jacq.) Müll. Arg. EAPG 2035

Plumeria rubra L. f. acutifolia (Poir.) Woodson CGH 1527, 1565, JM 1793, 2150

Prestonia mexicana A DC. EAPG 1901

Rauvolfia tetraphylla L. JM 1927, EAPG 930, 1096, 1741

* Stemmadenia eubracteata Woodson CGH 1912, EAPG 1482

$\begin{array}{ll} & x \\ x & X \\ X & x\end{array}$

$X$

$X$

$x$

$x$

$X$

$X$

$\mathrm{X}$

$\mathrm{X} \times$

X

$X$

X

$X$

Stemmadenia obovata (Hook. et Arn.)

K. Schum.

$x$

$X$ CGH 1461, 1568, 1922, EAPG 739, 1134, 1177, 1475

* Tabernaemontana alba Mill.

$X$

EAPG 1734 
Acta Botanica Mexicana (2001), 56: 19-88

Apéndice. Continuación.

Familias, Especies y Colectas

Tipo de vegetación

SAB SBC ME MX SM BG VA VS

* Tabernaemontana chrysocarpa S. F. Blake EAPG 1244

Thevetia plumeriifolia Benth.

CGH 1557, EAPG 856

ARISTOLOCHIACEAE

* Arsitolochia anguicida Jacq. X EAPG 2082

\section{ASCLEPIADACEAE}

Asclepias oenotheroides Cham. et Schltdl. $\mathrm{X} \quad \mathrm{X}$ CGH 1468, 2290, EAPG 1543

* Cynanchum racemosum (Jaq.) Jacq. var. unifarium (Scheele) Sundell

EAPG 1214, 1831

Gonolobus barbatus Kunth

CGH 1695, 2317, EAPG 973, 1010, 1228

* Macroscepis diademata (Ker Gawl.) W. D. Stevens CGH 1608, 1926, 2289, EAPG 1009

Marsdenia coulteri Hemsl.

CGH 1596, JM 1914, EAPG 829, 853, 970, 1107, 1137, 1724

Marsdenia mexicana Decne.

EAPG 1804

* Marsdenia gallardoae Lozada-Pérez EAPG 1504, 1625

* Marsdenia zimapanica Hemsl. EAPG 1114, 1229

* Matelea crenata (Vail) Woodson CGH 1696

Matelea cyclophylla (Standl.) Woodson X CGH 1430, JM 1768, EAPG 877

Matelea rupestris (Brandegee) Woodson X JM 2323, EAPG 1503

* Metastelma lanceolatum Schltr. X LLO 52, EAPG 1770

* Metastelma macropodum Greenm. X CGH 2276

$$
X
$$$$
\text { X } \quad X
$$

$X$

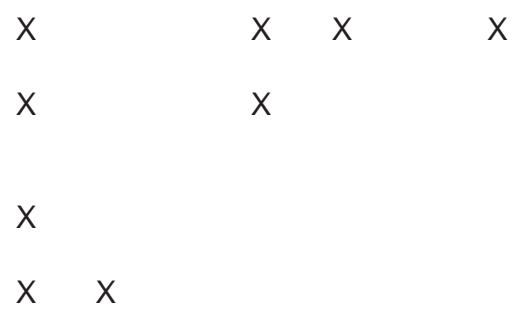

$X$

$X$

$X$ 
Apéndice. Continuación.

Familias, Especies y Colectas

Tipo de vegetación

SAB SBC ME MX SM BG VA VS

* Metastelma multiflorum S. Watson

$\mathrm{X}$

CGH 1936, 1975, 2292, 2302, JM 2127

* Sarcostemma bilobum Hook. et Arn.

EAPG 2013, 2038

Sarcostemma clausum (Jacq.) Schult.

$X$

EAPG 1755

\section{ASTERACEAE}

* Acourtia sp. nov. ined.

EAPG 1438

Adenophyllum aurantium (L.) Strother EAPG 1418, 1420, 1728

* Ageratina crassiramea (B. L. Rob.) R. M.

King et $\mathrm{H}$. Rob.

EAPG 1079

* Ageratum microcephalum Hemsl. X CGH 2308

* Baccharis salicifolia (Ruiz et Pav.) Pers. EAPG 1638

$X$

$\mathrm{X}$

$X$

Bidens squarrosa Kunth

EAPG 1364, 1570, 1667

Brickellia diffusa (Vahl) A. Gray

EAPG 880, 1682

Calea urticifolia (Mill.) DC. var. urticifolia X $\quad$ X

EAPG 1582, 1669

* Chromolaena breedlovei R. M. King et

H. Rob.

$X$

EAPG 1694

Chromolaena collina (DC.) R. M. King et H. Rob.

JM 2379, EAPG 1356

Chromolaena odorata (L.) R. M. King et

H. Rob.

EAPG 921

* Eclipta prostrata (L.) L.

EAPG 1756

* Espejoa mexicana DC.

$\mathrm{X} \times$

$X$

EAPG 1833 
Apéndice. Continuación.

Familias, Especies y Colectas

Tipo de vegetación

SAB SBC ME MX SM BG VA VS

Fleischmannia pycnocephala (Less.) R. M. King et $\mathrm{H}$. Rob.

$X$

EAPG 1415

Koanophyllon solidaginoides (Kunth) R. M. King et $\mathrm{H}$. Rob.

JM 1804, EAPG 737

Lasianthaea fruticosa (L.) K. M. Becker

var. fruticosa

JM 1824, 1868

* Melampodium sericeum Lag.

CGH 2286, LLO 60, JM 2344,

Milleria quinqueflora $\mathrm{L}$.

ELT 63

Montanoa tomentosa Cerv. subsp. microcephala (Sch. Bip.) V. A. Funk EAPG 789, 857, 1350

Parthenium hysterophorus L. EAPG 1556

*Pectis linearis La Llave X LLO 63

Pectis saturejoides (Mill.) Sch. Bip.

CGH 1974, 2275, JM 2124, EAPG 1328, 1397, 1409

Perymenium grande Hemsl. var. nelsonii (B. L. Rob. et Greenm.) J. J. Fay CGH 2257, EAPG 1662

* Perymenium aff. grande Hemsl. JM 2364

* Pittocaulon velatum (Greenm.) H. Rob. et Brettell var. tzimolense (T. M. Barkley) B. L. Clark CGH 1477, EAPG 1385

* Porophyllum macrocephalum DC. EAPG 1620

Porophyllum punctatum (Mill.) S. F. Blake $\quad X \quad X \quad X$ CGH 2280, JM 1787, EAPG 1608, 1685

Roldana eriophylla (Greenm.) H. Rob. et Brettell CGH 1487, EAPG 959

$X$ 
Apéndice. Continuación.

Familias, Especies y Colectas

Tipo de vegetación

SAB SBC ME MX SM BG VA VS

Simsia villasenorii D. M. Spooner

LLO 59, JM 2271

$X$

Sinclairia andrieuxii (DC.) H. Rob. et

Brettell

CGH 1598

$\mathrm{X}$

* Stenocephalum jucundum (Gleason) H.

Rob.

EAPG 1564

* Stevia connata Lag.

EAPG 1843

Tithonia tubiformis (Jacq.) Cass.

EAPG 745

Tridax procumbens $\mathrm{L}$.

JM 1861, EAPG 1425, 1545

Trixis inula Crantz

EAPG 1422

Verbesina abscondita Klatt $\quad X$

EAPG 799

Verbesina oaxacana DC. X

CGH 2261

*Verbesina persicifolia DC. X

EAPG 1837

Vernonanthura oaxacana (Sch. Bip. ex Klatt) H. Rob.

$X$

EAPG 912

*Viguiera tenuis A. Gray X

EAPG 1636

*Wamalchitamia strigosa (DC.) Strother $\quad X$

ELT 37

Wedelia acapulcensis Kunth var. tehuantepecana (B. L. Turner) Strother

CGH 2274, EAPG 714, 1524, 1566

Zinnia flavicoma (DC.) Olorode et A. M.

Torres

JM 2349

Zinnia cf. peruviana (L.) L. X

LLO 98 
Acta Botanica Mexicana (2001), 56: 19-88

Apéndice. Continuación.

Familias, Especies y Colectas

Tipo de vegetación

SAB SBC ME MX SM BG VA VS

BASELLACEAE

Anredera vesicaria (Lam.) C. F. Gaertn.

$X$

EAPG 1650

BIGNONIACEAE

Adenocalymma inundatum C. Mart. ex DC. CGH 1647, JM 1760, 2327, EAPG 1103

Amphilophium paniculatum (L.) Kunth CGH 1495, 1550

* Anemopaegma chrysanthum Dugand CGH 1909

* Arrabidaea costaricensis (Kränzl.) A. H. Gentry CGH 1599, 1600, 1614, 2352, EAPG

761, 812, 1175

Arrabidaea floribunda (Kunth) Loes.

CGH 1555, 1618, 2341, 2351, JM 1832

Arrabidaea patellifera (Schltdl.) Sandw.

CGH 2362, JM 1794, EAPG 1342, 1599

Astianthus viminalis (Kunth) Baill.

ELT 55, JM 2132, EAPG 1007

Crescentia alata Kunth

CGH 2313

Cydista potosina (K. Schum. et Loes.)

Loes.

$X$

$X$

$X$

$X$

$x$

EAPG 1341

* Godmania aesculifolia (Kunth) Standl.

EAPG 1693

Mansoa hymenaea (DC.) A. H. Gentry X

EAPG 939

* Mansoa verrucifera (Schltdl.) A. H. Gentry X

EAPG 1558

* Melloa quadrivalvis (Jacq.) A. H. Gentry X

EAPG 1498

Pithecoctenium crucigerum (L.) A. H.

Gentry

JM 1859, EAPG 927

Tabebuia chrysantha (Jacq.) G. Nicholson

EAPG 1053

X

$X$

X

X

X

X $x$

$x$

X $\quad X$

X 
Apéndice. Continuación.

\begin{tabular}{|c|c|c|c|c|c|c|c|c|}
\hline \multirow[t]{2}{*}{ Familias, Especies y Colectas } & \multicolumn{8}{|c|}{ Tipo de vegetación } \\
\hline & SAB & SBC & ME & $\mathrm{MX}$ & SM & $B G$ & VA & VS \\
\hline \multicolumn{9}{|l|}{ Tabebuia impetiginosa (Mart. ex DC.) } \\
\hline Standl. & & $\mathrm{X}$ & & & & & & \\
\hline CGH 2356, EAPG 734 & & & & & & & & \\
\hline $\begin{array}{l}\text { * Tabebuia pentaphylla (L.) Hemsl. } \\
\text { EAPG } 1444\end{array}$ & & & & & $x$ & & & \\
\hline $\begin{array}{l}\text { Tecoma stans (L.) Kunth } \\
\text { JM } 1891\end{array}$ & & & & $\mathrm{X}$ & & & & \\
\hline \multicolumn{9}{|l|}{ BIXACEAE } \\
\hline $\begin{array}{l}\text { Cochlospermum vitifolium (Willd.) Spreng. } \\
\text { CGH } 1650\end{array}$ & & $x$ & & & & & & \\
\hline \multicolumn{9}{|l|}{ BOMBACACEAE } \\
\hline $\begin{array}{l}\text { Ceiba parvifolia Rose } \\
\text { EAPG } 944,1088\end{array}$ & & $x$ & & & & & & \\
\hline $\begin{array}{l}\text { * Ceiba grandiflora Bartlett } \\
\text { CGH } 1885\end{array}$ & & $x$ & & & & & & \\
\hline $\begin{array}{l}\text { Pseudobombax ellipticum (Kunth) Dugand } \\
\text { CGH 1980, EAPG } 1029\end{array}$ & & $\mathrm{X}$ & & & & & & \\
\hline \multicolumn{9}{|l|}{ BORAGINACEAE } \\
\hline $\begin{array}{l}\text { Bourreria aff. andrieuxii (DC.) Hemsl. } \\
\text { EAPG } 1314\end{array}$ & & $\mathrm{X}$ & & & & & & \\
\hline $\begin{array}{l}\text { (*) Bourreria purpusii Brandegee }^{*} \text { CGH 1552, 1856, 1867, EAPG 1118, } \\
1203\end{array}$ & & $x$ & & & & & & \\
\hline Cordia curassavica (Jacq.) Roem. et & & & & & & & & \\
\hline $\begin{array}{l}\text { Schult. } \\
\text { JM 1792, 1854, 2314, EAPG } 715\end{array}$ & $x$ & $x$ & & & $x$ & & & \\
\hline $\begin{array}{l}\text { Cordia dentata Poir. } \\
\text { CGH 1694, JM 1771, } 1902\end{array}$ & & $x$ & & & $\mathrm{X}$ & & & \\
\hline $\begin{array}{l}\text { Cordia gerascanthus L. } \\
\text { EAPG } 964\end{array}$ & & $x$ & & & & & & \\
\hline $\begin{array}{l}\text { Cordia inermis (Mill.) I. M. Johnst. } \\
\text { CGH 1584, EAPG } 1480\end{array}$ & & $x$ & & & $\mathrm{X}$ & & & \\
\hline $\begin{array}{l}\text { Cordia oaxacana DC. } \\
\text { CGH 1609, EAPG 897, 1144, } 1606\end{array}$ & & $x$ & $\mathrm{X}$ & & & & & \\
\hline $\begin{array}{l}\text { Cordia truncatifolia Bartlett } \\
\text { JM 2146, 2281, EAPG 1052, 1302, } \\
1304\end{array}$ & $x$ & $x$ & & & $\mathrm{x}$ & & & \\
\hline
\end{tabular}


Apéndice. Continuación.

\begin{tabular}{|c|c|c|c|c|c|c|c|c|}
\hline \multirow[t]{2}{*}{ Familias, Especies y Colectas } & \multicolumn{8}{|c|}{ Tipo de vegetación } \\
\hline & $S A B$ & SBC & ME & $M X$ & SM & $B G$ & VA & VS \\
\hline $\begin{array}{l}\text { Heliotropium macrostachyum (DC.) Hemsl. } \\
\text { CGH 2320, JM 1840, } 2351\end{array}$ & & $x$ & & & $x$ & & & \\
\hline $\begin{array}{l}\text { Heliotropium procumbens Mill. } \\
\text { EAPG } 1299\end{array}$ & & & & & $\mathrm{x}$ & & & \\
\hline $\begin{array}{l}\text { Heliotropium ternatum Vahl } \\
\text { CGH 1934, JM 1797, 2111, 2153, AOP } \\
\text { 220, EAPG 1506, } 1591\end{array}$ & $\mathrm{x}$ & & $\mathrm{x}$ & & & & & \\
\hline $\begin{array}{l}\text { Tournefortia volubilis L. } \\
\text { EAPG } 961\end{array}$ & & $\mathrm{X}$ & & & & & & \\
\hline BURSERACEAE & & & & & & & & \\
\hline $\begin{array}{l}\text { * Bursera arborea (Rose) L. Riley } \\
\text { JM 1865, EAPG } 868\end{array}$ & & $x$ & & & $x$ & & & \\
\hline $\begin{array}{l}\text { Bursera bicolor (Willd. ex Schltdl.) Engl. } \\
\text { EAPG } 1192\end{array}$ & & & & $x$ & & & & \\
\hline $\begin{array}{l}\text { Bursera excelsa (Kunth) Engl. } \\
\text { JM 1764, 1765, 1878, EAPG 1163, } \\
1368\end{array}$ & & $\mathrm{x}$ & & & & & & \\
\hline $\begin{array}{l}\text { Bursera grandifolia (Schltdl.) Engl. } \\
\text { CGH 1677, JM } 1863\end{array}$ & & $\mathrm{x}$ & & & & & & \\
\hline $\begin{array}{l}\text { Bursera schlechtendalii Engl. } \\
\text { CGH 1520, 1528, 1564, JM 1763, 1880, } \\
\text { 2155, EAPG 1046, } 1083\end{array}$ & & $x$ & & $x$ & & & & \\
\hline BUXACEAE & & & & & & & & \\
\hline $\begin{array}{l}\text { * Buxus bartlettii Standl. } \\
\text { JM } 2375\end{array}$ & & $x$ & & & & & & \\
\hline CACTACEAE & & & & & & & & \\
\hline $\begin{array}{l}\text { * Acanthocereus horridus Britton et Rose } \\
\text { JM } 1876 \\
\text { * Cephalocereus nizandensis (Bravo et T }\end{array}$ & & $x$ & & & & & & \\
\hline $\begin{array}{l}\text { MacDoug.) Buxb. } \\
\text { CGH } 1531\end{array}$ & & & & $x$ & & & & \\
\hline $\begin{array}{l}\text { Epiphyllum phyllanthus (L.) Haw. var. } \\
\text { hookeri (Haw.) Kimnach } \\
\text { EAPG } 1335\end{array}$ & & $\mathrm{x}$ & & & & & & \\
\hline
\end{tabular}


Apéndice. Continuación.

Familias, Especies y Colectas

Tipo de vegetación

SAB SBC ME MX SM BG VA VS

Hylocereus undatus (Haw.) Britton et

Rose

$X$

EAPG 1132

* Mammillaria albilanata Backeb. EAPG 878

* Mammillaria voburnensis Scheer var. collinsii (Britton et Rose) Repp.

JM 1874, EAPG 798

Melocactus ruestii K. Schum.

EAPG 1502

* Neobuxbaumia scoparia (Poselg.) Backeb. CGH 1591

Nopalea karwinskiana (Salm-Dyck) K.

Schum.

CGH 1570

Nyctocereus oaxacensis Britton et Rose $X$ JM 2106, EAPG 1232

Opuntia puberula Pfeiff.

EAPG 2042

Pachycereus pecten-aboriginum (A.

Berger) Britton et Rose

EAPG 1441

* Peniocereus fosterianus Cutak var. nizandensis Sánchez-Mej.

CGH 1839, EAPG 1171, 1217, 1810

Pereskia lychnidiflora DC. JM 2157

Pereskiopsis kellermannii Rose EAPG 957

Pilosocereus collinsii (Britton et Rose)

Byles et G. D. Rowley

CGH 1590, EAPG 1319

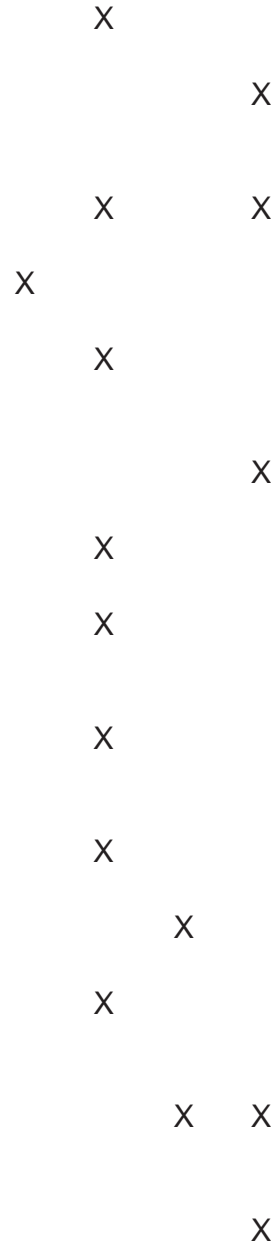

et Rose

EAPG 1218

* Selenicereus testudo (Karw. ex Zucc.)

Buxb.

$X$

CGH 1897 
Acta Botanica Mexicana (2001), 56: 19-88

Apéndice. Continuación.

Familias, Especies y Colectas

Tipo de vegetación

SAB SBC ME MX SM BG VA VS

CAESALPINIACEAE

Bauhinia divaricata L. CGH 1628

Bauhinia seleriana Harms CGH 1534, 1616, 1881, JM 1941

Caesalpinia coriaria (Jacq.) Willd. EAPG 1230, 1330

Caesalpinia eriostachys Benth. EAPG 1003

Caesalpinia exostemma DC. EAPG 936, 960

Caesalpinia platyloba S. Watson CGH 1502, 1545, EAPG 1775, 1886

$x$

Caesalpinia pulcherrima (L.) Sw. LLO 92

Caesalpinia sclerocarpa Standl. CGH 1905, JM 2322, EAPG 1547

* Chamaecrista diphylla (L.) Greene X LLO 84, EAPG 1583, 1690

Chamaecrista flexuosa (L.) Greene EAPG 1394, 1510

Chamaecrista hispidula (Vahl) H. S. Irwin et Barneby AOP 213, EAPG 1061, 1688

Chamaecrista nictitans (L) Moench var. jaliscensis (Greenm.) H. S. Irwin et Barneby LLO 77

Chamaecrista serpens (L.) Greene JM 2142, EAPG 1515

* Cynometra oaxacana Brandegee JM 1893, 2303, EAPG 790, 863

* Hymenaea courbaril L. JM 2309

Parkinsonia aculeata L. EAPG 1311

Poeppigia procera C. Presl $X$

$X$

$x$

$X$

$X$

$x$

$x$

CGH 2358, EAPG 1786

$\mathrm{X}$

$X$

$x$


Apéndice. Continuación.

\begin{tabular}{|c|c|c|c|c|c|c|c|}
\hline \multirow[t]{2}{*}{ Familias, Especies y Colectas } & \multicolumn{7}{|c|}{ Tipo de vegetación } \\
\hline & SAB SBC & ME & MX & SM & $B G$ & VA & VS \\
\hline $\begin{array}{l}\text { Senna alata (L.) Roxb. } \\
\text { CGH } 1997\end{array}$ & & & & $x$ & & & \\
\hline $\begin{array}{l}\text { Senna atomaria (L.) H. S. Irwin et } \\
\text { Barneby } \\
\text { CGH 1424, JM 1781, 2280, EAPG } 758 \text {, } \\
801,989\end{array}$ & $\mathrm{x}$ & & & $\mathrm{X}$ & & & \\
\hline $\begin{array}{l}\text { Senna fruticosa (Mill.) H. S. Irwin et } \\
\text { Barneby } \\
\text { CGH 1503, 1882, EAPG } 807\end{array}$ & $\mathrm{x}$ & & & & & & \\
\hline $\begin{array}{l}\text { Senna holwayana (Rose) H. S. Irwin et } \\
\text { Barneby } \\
\text { EAPG 899, } 1657\end{array}$ & $x$ & & & & $\mathrm{X}$ & & \\
\hline $\begin{array}{l}\text { Senna pallida (Vahl) H. S. Irwin et } \\
\text { Barneby } \\
\text { EAPG } 1842\end{array}$ & $\mathrm{x}$ & & & & & & \\
\hline $\begin{array}{l}\text { Senna uniflora (Mill.) H. S. Irwin et } \\
\text { Barneby } \\
\text { EAPG } 1557\end{array}$ & $\mathrm{x}$ & & & & & & \\
\hline CAPPARACEAE & & & & & & & \\
\hline $\begin{array}{l}\text { * Capparis baducca L. } \\
\text { JM 2108, EAPG } 1069\end{array}$ & $x$ & & & & & & \\
\hline $\begin{array}{l}\text { Capparis incana Kunth } \\
\text { CGH 1466, 1686, EAPG 1090, 1104, } \\
1120\end{array}$ & $\mathrm{X}$ & & & $\mathrm{X}$ & & & \\
\hline $\begin{array}{l}\text { Capparis indica (L.) Druce } \\
\text { CGH 1540, JM 2294, EAPG } 1067\end{array}$ & $x$ & & & $x$ & & & \\
\hline $\begin{array}{l}{ }^{*} \text { Capparis verrucosa Jacq. } \\
\text { EAPG 1042, 1072, } 1465\end{array}$ & $x$ & & & $\mathrm{X}$ & & & \\
\hline $\begin{array}{l}\text { Crateva tapia L. } \\
\text { CGH 1456, EAPG 1100, } 1473\end{array}$ & & & & $\mathrm{x}$ & $\mathrm{X}$ & & \\
\hline $\begin{array}{l}\text { Forchhammeria pallida Liebm. } \\
\text { EAPG } 874,875,888,1379,1472\end{array}$ & $x$ & & & $\mathrm{x}$ & & & \\
\hline $\begin{array}{l}\text { Gynandropsis speciosa (Raf.) DC. } \\
\text { JM 1899, EAPG } 766\end{array}$ & $x$ & & & & & & \\
\hline $\begin{array}{l}\text { Morisonia americana L. } \\
\text { JM 1754, EAPG } 1358\end{array}$ & $\mathrm{x}$ & & & $\mathrm{x}$ & & & \\
\hline $\begin{array}{l}\text { Polanisia viscosa (L.) DC. } \\
\text { CGH 1658, JM 1842, } 1926\end{array}$ & $x$ & & $x$ & & & & \\
\hline
\end{tabular}


Acta Botanica Mexicana (2001), 56: 19-88

Apéndice. Continuación.

Familias, Especies y Colectas

Tipo de vegetación

SAB SBC ME MX SM BG VA VS

CARICACEAE

Jacaratia mexicana A. DC.

EAPG 819, 845, 969, 971

$X$

CECROPIACEAE

* Cecropia peltata L. JM 2300, EAPG 1637

$X$

CELASTRACEAE

Crossopetalum uragoga (Jacq.) Kuntze X

EAPG 772, 994

* Rhacoma oxyphylla (S.F.Blake) Standl. X CGH 2328

* Rhacoma scoparia Standl. EAPG 1093

* Schaefferia frutescens Jacq. X CGH 1546

* Wimmeria confusa Hemsl. EAPG 1675

CHRYSOBALANACEAE

Licania arborea Seem.

EAPG 1617, 1656

$X$

CLUSIACEAE

Calophyllum brasiliense Cambess. $\quad \mathrm{X}$

EAPG 1499

Rheedia edulis (Seem.) Planch. et Triana JM 1816, EAPG 1430, 1437

COMBRETACEAE

Bucida macrostachya Standl. CGH 1434, 1499

* Combretum decandrum Jacq. EAPG 988

Combretum farinosum Kunth

CGH 1501, CGH 1576

X

X

X 
Apéndice. Continuación.

Familias, Especies y Colectas

Tipo de vegetación

SAB SBC ME MX SM BG VA VS

CONNARACEAE

* Rourea glabra Kunth

$X$

EAPG 861

CONVOLVULACEAE

*Convolvulus nodiflorus Desr.

EAPG sn

Evolvulus alsinoides (L.) L.

LLO 97, EAPG 1509

* Evolvulus cardiophyllus Schltdl.

EAPG 1839

* Evolvulus sericeus Sw.

EAPG 1780

Ipomoea bracteata Cav.

ELT 1, EAPG 825, 854, 873

* Ipomoea capillacea (Kunth) G. Don

CGH 2304, EAPG 1848

Ipomoea hederifolia L.

EAPG F56

* Ipomoea lutea Hemsl.

JM 1918

Ipomoea minutiflora (M. Martens et

Galeotti) House

EAPG 1827

Ipomoea nil (L.) Roth

JM 1852, 1919, EAPG 1374

Ipomoea populina House

EAPG 842

* Ipomoea robinsonii House

JM 1850

* Ipomoea setosa Ker Gawl.

CGH 2355, EAPG 774, 792

* Ipomoea suaveolens (M. Martens et

Galeotti) Hemsl.

X X

$x$

$x$

$X$

$X$

CGH 1476, 1515

$\left(^{*}\right)$ Ipomoea trifida (Kunth) G. Don

ELT 75

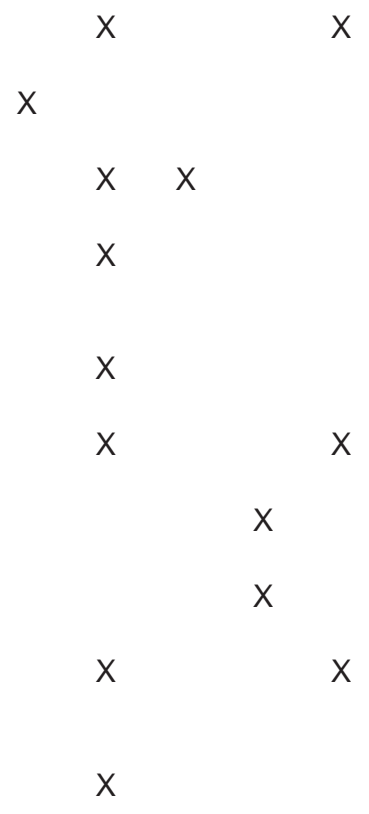


Acta Botanica Mexicana (2001), 56: 19-88

Apéndice. Continuación.

Familias, Especies y Colectas

Tipo de vegetación

SAB SBC ME MX SM BG VA VS

* Ipomoea variabilis (Schltdl. et Cham.)

Choisy

$X$

EAPG 986

* Jacquemontia mexicana (Loes.) Standl.

et Steyerm.

CGH 1646, 2327, EAPG 716

Merremia platyphylla (Fernald) O'Donell

CGH 1644, 2346, EAPG 833

* Merremia quinquefolia (L.) Hallier $f$.

EAPG 907

(*) Merremia umbellata (L.) Hallier

CGH 1622, 1639, 1657, EAPG 926

* Turbina corymbosa (L.) Raf.

EAPG 937, 993, 1737

CRASSULACEAE

Echeveria acutifolia Lindl.

CGH 1676, EAPG 872

CUCURBITACEAE

Cayaponia attenuata (Hook. et Arn.)

Cogn.

$X$

EAPG 1057

$\left({ }^{*}\right)$ Cayaponia racemosa (Mill.) Cogn. X

JM 2385

Doyerea emetocathartica Grosourdy X EAPG 1080

Ibervillea aff. hypoleuca (Standl.) C.

Jeffrey

CGH 1437, 1465, 1491, 1506, 1603,

1874, JM 2329, EAPG 1167

* Ibervillea millspaughii (Cogn.) C. Jeffrey EAPG 802

* Polyclathra albiflora (Cogn.) C. Jeffrey EAPG 1587

Polyclathra cucumerina Bertol. X

CGH 1673

* Rytidostylis gracilis Hook. et Arn. X

CGH 2338 
Apéndice. Continuación.

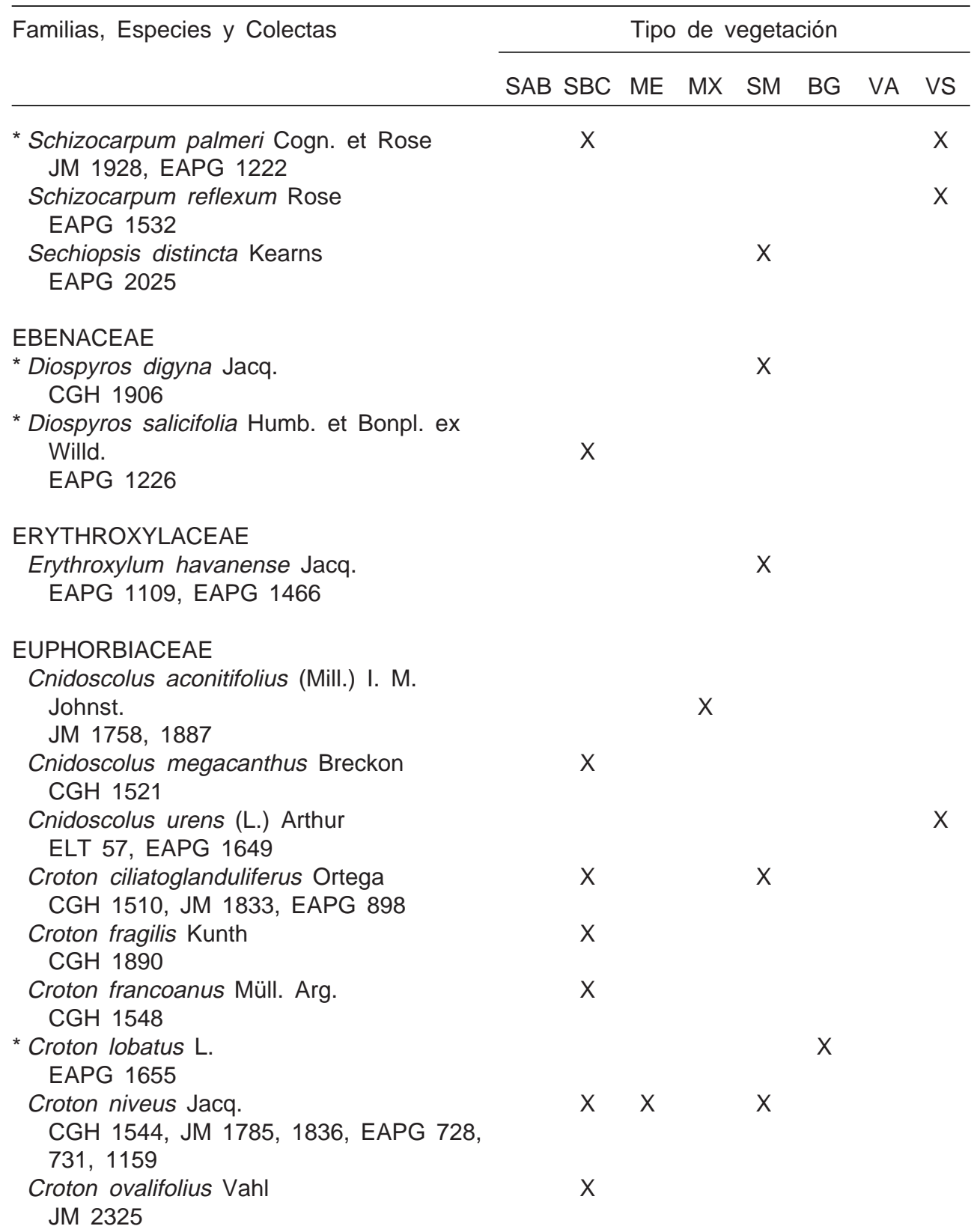


Acta Botanica Mexicana (2001), 56: 19-88

Apéndice. Continuación.

Familias, Especies y Colectas

Tipo de vegetación

SAB SBC ME MX SM BG VA VS

Croton pseudoniveus Lundell CGH 1872

* Croton yucatanensis Lundell EAPG 1867, 2121, 2123

Dalechampia scandens L. EAPG 749, 965

Ditaxis manzanilloana (Rose) Pax et K. Hoffm. CGH 2343

$X$

Euphorbia blodgetii Engelm. ex Hitchc. X LLO 99

* Euphorbia densiflora (Klotzsch et Garcke) Klotzsch EAPG 1962

Euphorbia heterophylla L. EAPG 1863

* Euphorbia hirta L. EAPG 1957

* Euphorbia pteroneura Berger EAPG 1431, 1746

Euphorbia schlechtendalii Boiss. EAPG 1411

Euphorbia segoviensis (Klotzsch et Garcke) Boiss.

EAPG 1959

* Hippomane mancinella L. CGH 1532, 1640, JM 1940, EAPG 722

Jatropha alamanii Müll. Arg. CGH 1542

Jatropha gossypiifolia L. CGH 1454

* Jatropha oaxacana J. Jiménez-Ram. et R. Torres CGH 1517, EAPG 1307, 1308

* Pedilanthus calcaratus Schlcht. X EAPG 1380

Pedilanthus tithymaloides (L.) Poit. subsp. tithymaloides

$X$

$\mathrm{X}$

$X$

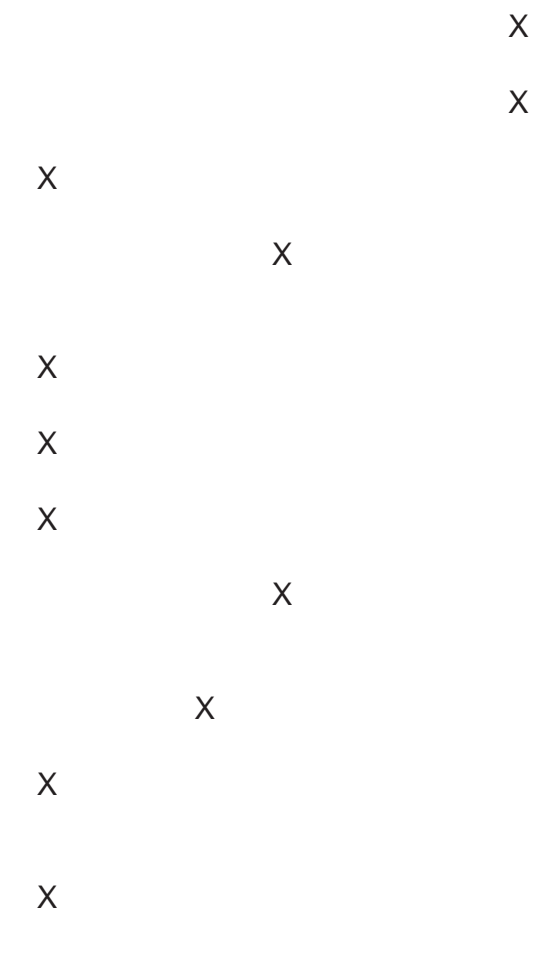


Apéndice. Continuación.

Familias, Especies y Colectas

Tipo de vegetación

SAB SBC ME MX SM BG VA VS

* Ricinus communis L. $X$ ELT 56

* Sapium glandulosum (L.) Morong JM 1894

* Sapium pedicellatum Huber CGH 1500, 1559

* Tragia mexicana Müll. Arg. CGH 1940, 2259, EAPG 717

FABACEAE

Aeschynomene compacta Rose

$X$

CGH 1551, EAPG 756, 800

Aeschynomene paniculata Willd. ex Vogel $\mathrm{X}$ EAPG 1584

Aeschynomene pinetorum Brandegee X LLO 27

Aeschynomene purpusii Brandegee

EAPG 1037, 1117

Andira inermis (W. Wright) Kunth ex DC. EAPG 1014, 1017

Apoplanesia paniculata C. Presl CGH 1536, EAPG 1359

Canavalia brasiliensis Mart. ex Benth. CGH 1692

Canavalia villosa Benth. EAPG 788

Chaetocalyx scandens (L.) Urb. EAPG 803, 1679, 1829

Coursetia caribaea (Jacq.) Lavin EAPG 740, 1683

Coursetia glandulosa A. Gray EAPG 962

* Coursetia oaxacensis M. Sousa et Rudd X CGH 1478

Coursetia polyphylla Brandegee EAPG 1128

Crotalaria cajanifolia Kunth

$X$

X $\quad X$

ELT 6, EAPG 1681, 1857

$\mathrm{x}$

$x$ 
Acta Botanica Mexicana (2001), 56: 19-88

Apéndice. Continuación.

Familias, Especies y Colectas

Tipo de vegetación

SAB SBC ME MX SM BG VA VS

Crotalaria pumila Ortega

LLO 61, EAPG 1575, 1853

Crotalaria sagittalis L.

$\mathrm{X}$

EAPG 1576

Dalea carthagenensis (Jacq.) J. F. Macbr. CGH 2321, EAPG 882

Dalea zimapanica Schauer JM 2396

* Desmodium barbatum (L.) Benth. X LLO 83

Diphysa americana (Mill.) M. Sousa EAPG 805

Diphysa humilis Oerst. ex Benth et Oerst. X LLO 31

Diphysa puberulenta Rydb. EAPG 1445

Eriosema crinitum (Kunth) G. Don X AOP 209, EAPG 1578

* Eriosema diffusum (Kunth) G. Don X EAPG 1970

Erythrina lanata Rose CGH 1572, 1706, JM 1770, EAPG 846

Galactia argentea Brandegee EAPG 1525, 1782

Galactia striata (Jacq.) Urb. JM 2369

Gliricidia sepium (Jacq.) Steud. EAPG 940, 1001, 1403, 1660

* Indigofera lespedezioides Kunth CGH 2297, LLO 26

* Indigofera thibaudiana DC. CGH 1588, JM 1924, EAPG 743

Lonchocarpus emarginatus Pittier CGH 1529, 1638, JM 1888, 1896, EAPG 811, 1369

Lonchocarpus lanceolatus Benth. EAPG 834, 835, 1721, ELT 40

* Lonchocarpus longipedicellatus Pittier EAPG 1809

$X$

$X$

$X$

$X$

$X$

\section{$X$}

$X$

$X$

$\mathrm{X}$

$X$

$x$

$X$

$X$

$X$

$x$

X

X 
Apéndice. Continuación.

Familias, Especies y Colectas

Tipo de vegetación

SAB SBC ME MX SM BG VA VS

* Lonchocarpus sp. nov. ined. 1

$\mathrm{X}$

EAPG 1022

Lonchocarpus sp. nov. ined. 2 EAPG 1600

* Machaerium pittieri Macbr. EAPG 1815

Macroptilium atropurpureum (Moc. et Sessé ex DC.) Urb. AOP 214, EAPG 1855

Macroptilium gracile (Poepp. ex Benth.) Urb.

AOP 210, EAPG 1062, 1692

Macroptilium longipedunculatum (C.Mart. ex Benth.) Urb.

EAPG 1354

Myrospermum frutescens Jacq. CGH 1684, 1726

Nissolia fruticosa Jacq. CGH 2345, 2349

* Nissolia microptera Poir. EAPG 751, 966

Phaseolus microcarpus Mart. EAPG 781, 1865

* Phaseolus oligospermus Piper EAPG 2002

Poiretia punctata (Willd.) Desv. X JM 2407

Pterocarpus rohrii Vahl CGH 1987, 1889, LLO 36, EAPG 976, 1038, 1075

Rhynchosia minima (L.) DC. EAPG 768, 1866

* Sesbania emerus (Aubl.) Urb. ELT 73

* Stylosanthes guianensis (Aubl.) Sw. X CGH 2273

* Stylosanthes humilis Kunth X EAPG 1321

$x$

$X$

X

$X$

$x$

$X$

$X$

$X$

X

X

X

X

X

X

X

$$
\text { X }
$$


Acta Botanica Mexicana (2001), 56: 19-88

Apéndice. Continuación.

Familias, Especies y Colectas

Tipo de vegetación

SAB SBC ME MX SM BG VA VS

Stylosanthes viscosa (L.) Sw. JM 2402

Tephrosia cinerea (L.) Pers. AOP 218, EAPG 1507

Tephrosia nicaraguensis Oerst. AOP 212, EAPG 1783

Tephrosia nitens Benth. JM 1796, EAPG 1060

Vigna speciosa (Kunth) Verdc. JM 1870

* Zornia megistocarpa Mohlenbr. AOP 204, EAPG 1530

* Zornia reticulata Sm. EAPG 1586

\section{FLACOURTIACEAE}

Casearia nitida (L.) Jacq. JM 1803, 1841, EAPG 1474

X

$X$

$X$

$X$

$X$

$x$

$\mathrm{X}$

Casearia tremula (Griseb.) Griseb. ex C. Wright CGH 1538, 1575, 1846

Muntingia calabura L. EAPG 1018

* Xylosma intermedia (Seem.) Triana et Planch.

CGH 1667

FOUQUIERIACEAE

Fouquieria formosa Kunth JM 1883, 1884

GENTIANACEAE

Eustoma exaltatum (L.) Salisb.

EAPG 1300, 1375

X

X

GESNERIACEAE

Achimenes grandiflora (Schiede) DC.

CGH 1908, ELT 35

X

X 
Apéndice. Continuación.

\begin{tabular}{|c|c|c|c|c|c|c|c|c|}
\hline \multirow[t]{2}{*}{ Familias, Especies y Colectas } & \multicolumn{8}{|c|}{ Tipo de vegetación } \\
\hline & SAB & SBC & ME & MX & SM & $B G$ & VA & VS \\
\hline \multicolumn{9}{|l|}{ HERNANDIACEAE } \\
\hline $\begin{array}{l}\text { Gyrocarpus mocinnoi Espejo } \\
\text { CGH 1643, EAPG } 967\end{array}$ & & $\mathrm{X}$ & & & & & & \\
\hline \multicolumn{9}{|l|}{ HIPPOCRATEACEAE } \\
\hline $\begin{array}{l}\text { Hemiangium excelsum (Kunth) A. C. Sm. } \\
\text { CGH 1995, JM 1873, 1808, } 1909\end{array}$ & & $\mathrm{X}$ & & $\mathrm{X}$ & $\mathrm{X}$ & & & \\
\hline \multicolumn{9}{|l|}{ Pristimera celastroides (Kunth) A. } \\
\hline $\begin{array}{l}\text { C. Sm. } \\
\text { EAPG 1389, } 1902\end{array}$ & & $\mathrm{X}$ & & & & & & \\
\hline \multicolumn{9}{|l|}{$\begin{array}{l}\text { HYDROPHYLLACEAE } \\
\text { * Wigandia urens (Ruiz et Pav.) Kunth var. }\end{array}$} \\
\hline $\begin{array}{l}\text { *Wigandia urens (Ruiz et Pav.) Kunth var. } \\
\text { caracasana (Kunth) D. N. Gibson } \\
\text { EAPG } 1006\end{array}$ & & & & & & $\mathrm{X}$ & & \\
\hline \multicolumn{9}{|l|}{ JULIANIACEAE } \\
\hline \multicolumn{9}{|l|}{ Amphipterygium adstringens (Schltdl.) } \\
\hline $\begin{array}{l}\text { Standl. } \\
\text { CGH 1482, 1516, JM 1774, EAPG } 1313\end{array}$ & & $\mathrm{X}$ & & & & & & \\
\hline \multicolumn{9}{|l|}{ KRAMERIACEAE } \\
\hline $\begin{array}{l}\text { Krameria revoluta O. Berg } \\
\text { EAPG } 1320,1400\end{array}$ & $\mathrm{X}$ & & & & & & & \\
\hline \multicolumn{9}{|l|}{ LAMIACEAE } \\
\hline $\begin{array}{l}\text { Asterohyptis stellulata (Benth.) Epling } \\
\text { EAPG } 1048\end{array}$ & & & & & $\mathrm{X}$ & & & \\
\hline $\begin{array}{l}\text { * Hyptis tomentosa Poit. } \\
\text { LLO 39, EAPG } 1771\end{array}$ & $\mathrm{X}$ & & $x$ & & & & & \\
\hline $\begin{array}{l}\text { * Ocimum micranthum Willd. } \\
\text { JM } 2332\end{array}$ & & $\mathrm{X}$ & & & & & & \\
\hline LAURACEAE & & & & & & & & \\
\hline Nectandra salicifolia (Kunth) Nees & & & & & $\mathrm{X}$ & & & \\
\hline
\end{tabular}


Acta Botanica Mexicana (2001), 56: 19-88

Apéndice. Continuación.

Familias, Especies y Colectas

Tipo de vegetación

SAB SBC ME MX SM BG VA VS

LOASACEAE

* Gronovia scandens L.

EAPG 1189

Mentzelia aspera L.

JM 1879

LOGANIACEAE

* Spigelia anthelmia L.

LLO 51

LORANTHACEAE

* Cladocolea oligantha (Standl. et Steyermark) Kuijt

$X$

CGH 1604

* Psittacanthus schiedeanus (Schltdl. et

Cham.) Blume

JM 1907

* Struthanthus cassythoides Millsp. ex

Standl.

CGH 1493

MALPIGHIACEAE

* Banisteriopsis acapulcensis (Rose) Small CGH 1675, 2360

Bunchosia canescens DC.

JM 1834, 1929

* Bunchosia lindeniana A. Juss.

EAPG 764

Byrsonima crassifolia (L.) Kunth

CGH 1973, JM 1791, 2147

Gaudichaudia albida Schltdl. et Cham. X

CGH 2258, EAPG 1630

Heteropterys cotinifolia A. Juss.

CGH 1567, JM 1908, 1911

* Heteropterys laurifolia (L.) A. Juss.

EAPG 1076, 1110

* Hiraea reclinata Jacq.

CGH 1996, JM 2129, EAPG 1479

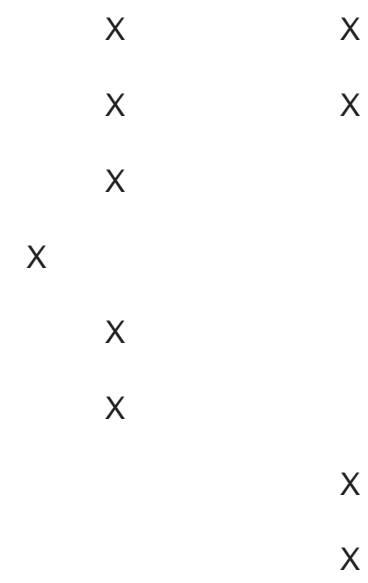


Apéndice. Continuación.

\begin{tabular}{|c|c|c|c|c|c|c|c|c|}
\hline \multirow{2}{*}{ Familias, Especies y Colectas } & \multicolumn{8}{|c|}{ Tipo de vegetación } \\
\hline & SAB & SBC & ME & $\mathrm{MX}$ & SM & $B G$ & VA & VS \\
\hline $\begin{array}{l}\text { Lasiocarpus salicifolius Liebm. } \\
\text { EAPG } 1032\end{array}$ & & $\mathrm{X}$ & & & & & & \\
\hline $\begin{array}{l}\text { Malpighia emarginata Sessé et Moc. } \\
\text { ex DC. } \\
\text { JM } 2136\end{array}$ & & $\mathrm{X}$ & & & & & & \\
\hline $\begin{array}{l}\text { Malpighia glabra L. } \\
\text { CGH 1664, EAPG } 793\end{array}$ & & & & & $\mathrm{X}$ & & & \\
\hline $\begin{array}{l}\text { Malpighia ovata Rose } \\
\text { CGH 1600, JM 1825, EAPG } 1758\end{array}$ & & $\mathrm{X}$ & & & & & & \\
\hline $\begin{array}{c}\text { Mascagnia dipholiphylla (Small) Bullock } \\
\text { CGH 1984, EAPG 1055, 1082, } 1391\end{array}$ & & $\mathrm{X}$ & & & & & & \\
\hline $\begin{array}{l}\text { * Stigmaphyllon lindenianum A. Juss. } \\
\text { CGH 1962, EAPG 987, } 1757\end{array}$ & & $\mathrm{X}$ & & & $x$ & & & \\
\hline Tetrapterys heterophylla (Griseb.) W. R. & & & & & & & & \\
\hline $\begin{array}{l}\text { Anderson } \\
\text { EAPG } 977\end{array}$ & & & & & $\mathrm{x}$ & & & \\
\hline $\begin{array}{l}\text { * Tetrapterys seleriana Nied. } \\
\text { JM 1786, EAPG 1073, } 1670\end{array}$ & & $\mathrm{X}$ & $\mathrm{X}$ & & & & & \\
\hline MALVACEAE & & & & & & & & \\
\hline $\begin{array}{l}\text { Abutilon barrancae M. E. Jones } \\
\text { EAPG } 755\end{array}$ & & $\mathrm{X}$ & & & & & & \\
\hline $\begin{array}{l}\text { * Abutilon haenkeanum C. Presl } \\
\text { EAPG } 1766\end{array}$ & & $\mathrm{X}$ & & & & & & \\
\hline $\begin{array}{l}\text { Herissantia crispa (L.) Brizicky } \\
\text { CGH 2348, EAPG } 1634\end{array}$ & $\mathrm{X}$ & & & & & & & \\
\hline $\begin{array}{l}\text { Hibiscus kochii Fryxell } \\
\text { EAPG 762, 771, 827, 879, } 1174\end{array}$ & & $\mathrm{X}$ & & & & & & \\
\hline $\begin{array}{l}\text { Hibiscus phoeniceus Jacq. } \\
\text { CGH 1663, EAPG 919, } 1122\end{array}$ & & $\mathrm{X}$ & & & $x$ & & & \\
\hline $\begin{array}{l}\text { Pavonia macdougallii Fryxell } \\
\text { CGH 2318, EAPG } 754\end{array}$ & & $\mathrm{X}$ & & & & & & \\
\hline $\begin{array}{l}\text { *Pavonia paniculata Cav. } \\
\text { EAPG } 925\end{array}$ & & & & & $x$ & & & \\
\hline MELIACEAE & & & & & & & & \\
\hline $\begin{array}{l}\text { * Cedrela salvadorensis Standl. } \\
\text { CGH 1907, JM 1822, EAPG } 12 \varsigma\end{array}$ & & $\mathrm{X}$ & & & & & & \\
\hline
\end{tabular}


Acta Botanica Mexicana (2001), 56: 19-88

Apéndice. Continuación.

Familias, Especies y Colectas

Tipo de vegetación

SAB SBC ME MX SM BG VA VS

Swietenia humilis Zucc.

EAPG 1231, 1635

Trichilia havanensis Jacq.

EAPG 1197

MENISPERMACEAE

Cocculus diversifolius DC.

EAPG 1008

Hyperbaena mexicana Miers

CGH 1990, 1991, JM 2282, EAPG

1023, 1094, 1485

MIMOSACEAE

Acacia cochliacantha Humb. et Bonpl.

ex Willd.

EAPG 752, 885, 1143

Acacia coulteri Benth.

CGH 1880, EAPG 1135

Acacia farnesiana (L.) Willd.

EAPG 886

Acacia aff. hindsii Benth.

EAPG 1739

Acacia picachensis Brandegee

CGH 1512, EAPG 725, 884, 1151, 1204

Acacia pringlei Rose

EAPG 997

Acacia tenuifolia (L.) Willd.

JM 1828

Acacia villosa (Sw.) Willd.

AOP 203, CGH 2295, LLO 38

$\mathrm{X}$

$\mathrm{X}$

$x$

Albizia guachapele (Kunth) Dugand EAPG 1035

Calliandra bijuga Rose

EAPG 1950

Calliandra juzepczukii Standl.

EAPG 1527

Calliandra tergemina (L.) Benth. var.

emarginata (Humb. et Bonpl. ex Willd.) X X

Barneby

CGH 1436, EAPG 1773 
Apéndice. Continuación.

\begin{tabular}{|c|c|c|c|c|c|c|c|c|}
\hline \multirow[t]{2}{*}{ Familias, Especies y Colectas } & \multicolumn{8}{|c|}{ Tipo de vegetación } \\
\hline & SAB & SBC & ME & $\mathrm{MX}$ & SM & BG & VA & VS \\
\hline $\begin{array}{l}\text { Chloroleucon mangense (Jacq.) Britton } \\
\text { et Rose } \\
\text { CGH 1993, EAPG } 1301\end{array}$ & & $\mathrm{X}$ & & & $X$ & & & \\
\hline $\begin{array}{l}\text { Desmanthus virgatus (L.) Willd. } \\
\text { EAPG } 1536\end{array}$ & & & & & & & & $\mathrm{X}$ \\
\hline $\begin{array}{l}\text { Enterolobium cyclocarpum (Jacq.) Griseb. } \\
\text { EAPG 946, } 1178\end{array}$ & & & & & $\mathrm{X}$ & & & \\
\hline Havardia campylacantha (L. Rico et M. & & & & & & & & \\
\hline $\begin{array}{l}\text { Sousa) Barneby et J. W. Grimes } \\
\text { CGH 1868, EAPG } 1155\end{array}$ & & $\mathrm{X}$ & & & $X$ & & & \\
\hline $\begin{array}{l}\text { Inga vera Willd. } \\
\text { EAPG 938, 1011, 1015, 1154, } 1198\end{array}$ & & & & & $\mathrm{X}$ & & & \\
\hline $\begin{array}{l}\text { Leucaena lanceolata S. Watson } \\
\text { CGH 1629, 1710, EAPG 742, 1569, } \\
1619\end{array}$ & & $\mathrm{X}$ & $x$ & & & & & \\
\hline $\begin{array}{l}\text { * Lysiloma divaricatum (Jacq.) J. F. Macbr. } \\
\text { CGH 1484, EAPG 757, 1241, } 1571\end{array}$ & $\mathrm{X}$ & $\mathrm{X}$ & & & & & & \\
\hline $\begin{array}{l}\text { * Lysiloma microphyllum Benth. } \\
\text { CGH 1688, JM 2319, EAPG } 1124\end{array}$ & & $\mathrm{X}$ & & & & & & \\
\hline Mimosa acantholoba (Humb. et Bonpl. ex & & & & & & & & \\
\hline $\begin{array}{l}\text { Willd.) Poir. var. eurycarpa B. L .Rob. } \\
\text { EAPG } 883\end{array}$ & & $\mathrm{X}$ & & & & & & \\
\hline $\begin{array}{l}\text { * Mimosa aculeaticarpa Ortega } \\
\text { EAPG } 1310\end{array}$ & & $\mathrm{X}$ & & & & & & \\
\hline $\begin{array}{l}\text { Mimosa albida Humb. et Bonpl. ex Willd } \\
\text { JM } 2393\end{array}$ & $x$ & & & & & & & \\
\hline $\begin{array}{l}\text { * Mimosa antioquiensis Killip ex Rudd var. } \\
\text { isthmensis R. Grether } \\
\text { EAPG 1516, 1528, 2361, JM } 1913\end{array}$ & $X$ & $\mathrm{X}$ & & & & & & \\
\hline $\begin{array}{l}\text { Mimosa goldmanii B. L. Rob. } \\
\text { CGH 2283, EAPG 753, 1306, } 1327\end{array}$ & $\mathrm{X}$ & $\mathrm{X}$ & $x$ & & & & & \\
\hline $\begin{array}{l}\text { Mimosa lactiflua Delile ex Benth. } \\
\text { CGH 2277, 2278, 2279, JM 2345, } \\
\text { AOP } 216\end{array}$ & $\mathrm{X}$ & & & & & & & \\
\hline $\begin{array}{l}\text { Mimosa mellii Britton et Rose } \\
\text { EAPG } 1668\end{array}$ & & $\mathrm{X}$ & & & & & & \\
\hline $\begin{array}{l}\text { * Mimosa skinneri Benth. } \\
\text { LLO } 65\end{array}$ & $\mathrm{X}$ & & & & & & & \\
\hline
\end{tabular}


Acta Botanica Mexicana (2001), 56: 19-88

Apéndice. Continuación.

Familias, Especies y Colectas

Tipo de vegetación

SAB SBC ME MX SM BG VA VS

Mimosa tenuiflora (Willd.) Poir.

$X$

EAPG 963

Piptadenia flava (Spreng. ex DC.) Benth. $\quad X$ CGH 1879

Piptadenia obliqua (Pers.) J. F. Macbr. CGH 2357, JM 1790, EAPG 724

Prosopis laevigata (Humb. et Bonpl. ex Willd.) M. C. Johnst.

EAPG 996

Zapoteca formosa (Kunth) H. M. Hern. subsp. rosei (Wiggins) H. M. Hern.

CGH 1615, 2264, EAPG 917, 1885

MORACEAE

Brosimum alicastrum Sw. CGH 1921, EAPG 1412

* Dorstenia contrajerva L. EAPG 1481

Dorstenia drakena L. X X CGH 1508

Ficus cotinifolia Kunth $\quad \mathrm{X}$ JM 1858

Ficus insipida Willd. CGH 1605, 1606

Ficus ovalis (Liebm.) Miq. CGH 1472, 1530, EAPG 786, 844

Ficus pertusa L. f. CGH 1504, 1642, JM 1846, EAPG 785

Ficus petiolaris Kunth CGH 1433, EAPG 1292, 1762

Ficus trigonata L. EAPG 1410

MORINGACEAE

Moringa oleifera Lam. LLO 40

$\mathrm{X} \quad \mathrm{X}$

$X$

$\mathrm{X}$

$X$

$X$

$X$

$x$

$x$<smiles>[X]</smiles>

$x$

$X \quad X$

$X$

$X$

MYRSINACEAE

(*) Ardisia paschalis Donn. Sm. CGH 1925, JM 1807, EAPG 1066, 1497

X $\quad X$ 
Apéndice. Continuación.

Familias, Especies y Colectas

Tipo de vegetación

SAB SBC ME MX SM BG VA VS

MYRTACEAE

* Calyptranthes schiedeana O. Berg

$X$

ELT 26, EAPG 1025, 1835

Eugenia aff. salamensis Donn. Sm. CGH 2314

* Psidium guineense Sw. EAPG 1687

* Psidium hypoglaucum Standl. X JM 2112

* Psidium salutare (Kunth) O. Berg X JM 2123

NYCTAGINACEAE

* Boerhavia coccinea Mill.

CGH 2336, EAPG 810, 1542

Boerhavia erecta L. JM 2330, EAPG 1344

* Grajalesia fasciculata (Standl.) Miranda EAPG 1044

* Mirabilis violacea (L.) Heimerl EAPG 1794

Pisonia aculeata L.

CGH 1537, 1602, JM 1944, EAPG 855,

X

$\mathrm{X}$ 1489

OCHNACEAE

Ouratea mexicana (Humb. et Bonpl.)

Engelm.

EAPG 1423

\section{$x$}

$X$

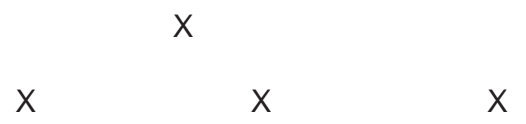

OLACACEAE

Ximenia americana $\mathrm{L}$.

X

EAPG 1081

\section{OLEACEAE}

* Forestiera aff. rhamnifolia Griseb. X EAPG 974

Fraxinus purpusii Brandegee

LLO 19, JM 2105 
Acta Botanica Mexicana (2001), 56: 19-88

Apéndice. Continuación.

Familias, Especies y Colectas

Tipo de vegetación

SAB SBC ME MX SM BG VA VS

ONAGRACEAE

Hauya elegans DC.

CGH 1435, 1569, JM 2343, EAPG 783

* Ludwigia erecta (L.) Itara CGH 1968

* Ludwigia octovalvis (Jacq.) P. H. Raven EAPG 1377

OXALIDACEAE

Oxalis neaei DC. EAPG 1477

* Oxalis pringlei (Rose) R. Knuth EAPG 1789

X $\mathrm{X}$

PAPAVERACEAE

Argemone mexicana L. EAPG 985

PASSIFLORACEAE

Passiflora filipes Benth. CGH 1617, 1620, EAPG 773

Passiflora foetida L.

EAPG 992

* Passiflora foetida var. hirsutissima Killip X JM 2306

* Passiflora mexicana Juss. CGH 1691, EAPG 972

* Passiflora suberosa L. CGH 2353

PEDALIACEAE

Martynia annua L. EAPG 1555

PHYTOLACCACEAE

Petiveria alliacea $\mathrm{L}$. JM 1813, EAPG 1353

Rivina humilis L. CGH 1660, 1837, 1901, ELT 43, EAPG 1348, 1432

X

$X$

$X$

$X$
X

X

X

X

X

X

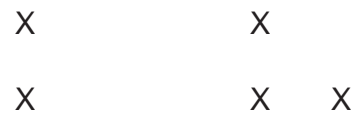


Apéndice. Continuación.

Familias, Especies y Colectas

Tipo de vegetación

SAB SBC ME MX SM BG VA VS

PIPERACEAE

Peperomia asarifolia Schltdl. et Cham. ELT 36, EAPG 1560, 1811

* Peperomia glutinosa Millsp. EAPG 1731

Piper auritum Kunth EAPG 1421

* Piper marginatum Jacq. JM 1821, EAPG 1490

Piper tuberculatum Jacq. EAPG 1469

PLUMBAGINACEAE

Plumbago scandens L. CGH 2336, EAPG 796, 866

$X$

$X$

POLYGALACEAE

* Polygala leptocaulis Torr. et Gray X LLO 100

*Polygala longicaulis Kunth X CGH 2311

* Polygala paniculata L. X X LLO 96

*Polygala serpens S. F. Blake X CGH 2269, EAPG 1643

Polygala variabilis Kunth $\mathrm{f}$. leucanthema S. F. Blake JM 1799, EAPG 1514

POLYGONACEAE

Antigonon cinerascens $\mathrm{M}$. Martens et Galeotti EAPG 1371

Antigonon flavescens S. Watson JM 1789

Coccoloba barbadensis Jacq. EAPG 1355

Coccoloba liebmannii Lindau CGH 1972, 2164, 2350, EAPG 1361

$X$

$X$

$X$

$X$

$X$

\section{$x$}

$x$

$x$

$x$

$x$

X 
Acta Botanica Mexicana (2001), 56: 19-88

Apéndice. Continuación.

Familias, Especies y Colectas

Tipo de vegetación

SAB SBC ME MX SM BG VA VS

Ruprechtia pallida Standl.

EAPG 1357

PORTULACACEAE

Portulaca oleracea L. X X

EAPG 1331

Portulaca pilosa L.

EAPG 1345

Talinum paniculatum (Jacq.) Gaertn.

ELT 48, JM 1826, EAPG 1795

Talinum triangulare (Jacq.) Willd.

CGH 1446, 1583, JM 1823, EAPG

$x$

1123

PRIMULACEAE

* Samolus ebracteatus Kunth

EAPG 1091, 1451

\section{RHAMNACEAE}

Colubrina elliptica (Sw.) Brizicky et Stern CGH 1535, 1639, 1682

Gouania lupuloides (L.) Urb.

CGH 1525, 2263, EAPG 1567, 1604

Gouania polygama (Jacq.) Urb.

CGH 1613, ELT 42

Karwinskia humboldtiana (Roem. et

Schult.) Zucc.

CGH 2344

* Krugiodendron ferreum (Vahl) Urb.

CGH 1523, 1978, 1994, JM 2156,

\section{$x$}

$X$<smiles>[X]</smiles>

$\mathrm{X}$

$x$ 2305, EAPG 1338

Ziziphus amole (Sessé et Moc.) M. C. Johnst.

CGH 1460, JM 1895, EAPG 1158 , 1239

\section{RUBIACEAE}

* Augusta rivalis (Benth.) J. H. Kirkbr.

$$
X
$$

EAPG 1115 
Apéndice. Continuación.

Familias, Especies y Colectas

Tipo de vegetación

SAB SBC ME MX SM BG VA VS

* Borreria suaveolens G. Mey. EAPG 2312

* Borreria verticillata (L.) G. Mey. LLO 23 JM 2314, EAPG 1592

* Calycophyllum candidissimum (Vahl) DC. CGH 1652

* Diodia rigida (Humb. et Bonpl. ex Kunth) Cham. et Schltdl.

LLO 42, EAPG 1593

$\mathrm{X}$

$X$

$x$

$X$

Diodia teres Walter

EAPG 1533

Exostema caribaeum (Jacq.) Roem. et Schult.

CGH 1507, 1690, EAPG 808

Exostema mexicanum A. Gray EAPG 1343

Genipa americana L. JM 2307, EAPG 1671

* Guettarda macrosperma Donn. Sm. JM 2291

Hamelia versicolor A. Gray CGH 1426, JM 1801, EAPG 1462

Hintonia latiflora (Sessé et Moc. ex DC.)

Bullock

EAPG 1627

* Psychotria horizontalis Sw. EAPG 1841

Randia aculeata L.

CGH 915, JM 1838, EAPG 741, 1173

* Randia nelsonii Greenm. CGH 1610

Randia thurberi S. Watson JM 1905, EAPG 787

Rondeletia deamii (Donn. Sm.) Standl. EAPG 1468

Rondeletia leucophylla Kunth EAPG 733, 1160, 1470

* Spermacoce confusa Rendle EAPG 1531
$X$

$X$

\section{$X$}

$X$

$x$

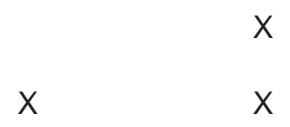

$x$

$\mathrm{X}$

X

X

X

X 
Acta Botanica Mexicana (2001), 56: 19-88

Apéndice. Continuación.

Familias, Especies y Colectas

Tipo de vegetación

SAB SBC ME MX SM BG VA VS

RUTACEAE

* Amyris aff. sylvatica Jacq. 1763

$X$ JM 1819

* Casimiroa tetrameria Millsp.

$X$ EAPG 1068, 1720

Esenbeckia berlandieri Baill. ex. Hemsl. subsp. litoralis (Donn. Sm.) Kaastra CGH 1611, JM 3221, EAPG 1624

Esenbeckia collina Brandegee CGH 1440, JM 1839, EAPG 836, 837, 887

Pilocarpus racemosus Vahl var. racemosus EAPG 838, 984, 726, 1735

* Zanthoxylum arborescens Rose CGH 1539, JM 1915, EAPG 759, 1300

Zanthoxylum fagara (L.) Sarg. ELT 24

$X$

$X$

$X$

SALICACEAE

Salix humboldtiana Willd.

EAPG 1816

SAPINDACEAE

* Cupania glabra Sw.

$x$ EAPG 1161

Paullinia cururu L. CGH 1429, JM 1802, 2277, EAPG 1196

* Paullinia pinnata L. JM 1946

Sapindus saponaria L. CGH 1630, EAPG 1754

* Serjania caracasana (Jacq.) Willd. CGH 1619, EAPG 903

Serjania grosii Schltdl. CGH 1524

Serjania triquetra Radlk. CGH 1573, JM 1890, 1932, EAPG 818

X 
Apéndice. Continuación.

\begin{tabular}{|c|c|c|c|c|c|c|c|c|}
\hline \multirow[t]{2}{*}{ Familias, Especies y Colectas } & \multicolumn{8}{|c|}{ Tipo de vegetación } \\
\hline & SAB & SBC & ME & $\mathrm{MX}$ & SM & $B G$ & VA & VS \\
\hline $\begin{array}{l}\text { * Thouinia acuminata S.Watson } \\
\text { CGH } 1659\end{array}$ & & & & & $\mathrm{X}$ & & & \\
\hline $\begin{array}{l}\text { * Thouinia villosa DC. } \\
\text { JM } 2138\end{array}$ & & $x$ & & & & & & \\
\hline $\begin{array}{l}\text { Thouinidium decandrum (Bonpl.) Radlk. } \\
\text { CGH 1967, ELT 12, 14, EAPG 1012, } \\
1095\end{array}$ & & & & & $\mathrm{x}$ & $x$ & & \\
\hline SAPOTACEAE & & & & & & & & \\
\hline $\begin{array}{l}\text { Manilkara zapota (L.) P. Royen } \\
\text { CGH 1469, EAPG } 1092\end{array}$ & & & & & $\mathrm{X}$ & & & \\
\hline $\begin{array}{l}\text { Sideroxylon capiri (A. DC.) Pittier } \\
\text { EAPG } 1024\end{array}$ & & & & & $\mathrm{X}$ & & & \\
\hline Sideroxylon celastrinum (Kunth) T. D. & & & & & & & & \\
\hline $\begin{array}{l}\text { Penn. } \\
\text { EAPG 978, } 1772\end{array}$ & & & $x$ & & & & & \\
\hline $\begin{array}{l}\text { * Sideroxylon obtusifolium (Roem. et Schult.) } \\
\text { T. D. Penn. subsp. buxifolium (Roem. }\end{array}$ & & & & & & & & \\
\hline $\begin{array}{l}\text { et Schult.) T. D. Penn. } \\
\text { CGH 1444, 1989, ELT } 10 \text { JM 1800, } \\
\text { 2290, EAPG } 1112\end{array}$ & & $x$ & & & $\mathrm{x}$ & & & \\
\hline $\begin{array}{l}\text { Sideroxylon stenospermum (Standl.) T. D. } \\
\text { Penn. } \\
\text { CGH 1566, EAPG } 1768\end{array}$ & & $\mathrm{X}$ & & & & & & \\
\hline SCROPHULARIACEAE & & & & & & & & \\
\hline $\begin{array}{l}\text { Bacopa monnieri (L.) Wettst. } \\
\text { EAPG } 1002\end{array}$ & & $\mathrm{X}$ & & & & & & \\
\hline $\begin{array}{l}\text { Buchnera pusilla Kunth } \\
\text { CGH 2342, JM 2395, AOP 206, EAPG } \\
1562\end{array}$ & $\mathrm{x}$ & & & & & & & \\
\hline $\begin{array}{l}\text { Capraria biflora L. } \\
\text { CGH } 2322\end{array}$ & & $x$ & & & & & & \\
\hline $\begin{array}{l}\text { Lamourouxia viscosa Kunth } \\
\text { CGH 2299, LLO 30, EAPG } 1588\end{array}$ & $x$ & & & & & & & \\
\hline $\begin{array}{l}\text { Russelia coccinea (L) Wettst. } \\
\text { EAPG 1732, } 2023\end{array}$ & & & & & $x$ & & & \\
\hline $\begin{array}{l}\text { * Russelia obtusata Blake } \\
\text { EAPG } 2031\end{array}$ & $\mathrm{X}$ & & & & & & & \\
\hline
\end{tabular}


Acta Botanica Mexicana (2001), 56: 19-88

Apéndice. Continuación.

Familias, Especies y Colectas

Tipo de vegetación

SAB SBC ME MX SM BG VA VS

* Russelia polyedra Zucc.

EAPG 1872

Russelia retrorsa Greene X

EAPG 1968

Russelia rugosa Robinson

CGH 2267, EAPG 1324

Schistophragma pusilla Benth.

LLO 62, JM 1912, EAPG 1512, 1850

Stemodia durantifolia (L.) Sw.

JM 2367

SIMAROUBACEAE

Castela retusa Liebm.

JM 2371

Recchia connaroides (Loes. et Soler)

Standl.

CGH 1680, 1707, 1976, 1979, EAPG

X

948, 954, 1258

\section{SOLANACEAE}

Capsicum annuum L. var. glabriusculum (Dunal) Heiser et Pickersgill

CGH 1869, JM 1837, 2350, EAPG 920

* Datura inoxia Mill.

EAPG 1653

Juanulloa mexicana (Schltld.) Miers

EAPG 1433

* Nicotiana plumbaginifolia Viv. X

CGH 1970

* Physalis arborescens L. CGH 1580, 2325, JM 1831, EAPG 738, 760, 839, 1105, 1546

Physalis maxima Mill. EAPG 1818

Physalis solanacea (Schltdl.) Axelius

ELT 66

Solandra nizandensis Matuda

X

X

CGH 2316 
Apéndice. Continuación.

\begin{tabular}{|c|c|c|c|c|c|c|c|c|}
\hline \multirow[t]{2}{*}{ Familias, Especies y Colectas } & \multicolumn{8}{|c|}{ Tipo de vegetación } \\
\hline & SAB & SBC & ME & MX & SM & $B G$ & VA & VS \\
\hline $\begin{array}{l}\text { Solanum americanum Mill. } \\
\text { EAPG } 991\end{array}$ & & $x$ & & & & & & \\
\hline $\begin{array}{l}\text { * Solanum diphyllum L. } \\
\text { JM 2286, EAPG 1106, 1298, } 1641\end{array}$ & & & & & $x$ & & & \\
\hline $\begin{array}{l}\text { Solanum glaucescens Zucc. } \\
\text { CGH 1464, 1562, 1876, JM 1925, } \\
\text { 1945, EAPG } 1149\end{array}$ & & $x$ & & & & & & \\
\hline $\begin{array}{l}\text { Solanum hazenii Britton } \\
\text { CGH 1665, JM 2275, 2308, EAPG } 778\end{array}$ & & $x$ & & & & & & \\
\hline STERCULIACEAE & & & & & & & & \\
\hline $\begin{array}{l}\text { * Ayenia glabra S. Watson } \\
\text { CGH } 1585\end{array}$ & & $x$ & & & & & & \\
\hline $\begin{array}{l}\text { Ayenia micrantha Standl. } \\
\text { CGH } 1857\end{array}$ & & $x$ & & & & & & \\
\hline $\begin{array}{l}\text { Byttneria aculeata (Jacq.) Jacq. } \\
\text { CGH } 1697\end{array}$ & & & & & $\mathrm{X}$ & & & \\
\hline $\begin{array}{l}\text { Guazuma ulmifolia Lam. } \\
\text { CGH 1653, EAPG 1101, } 1613\end{array}$ & & & & & $x$ & $x$ & & $x$ \\
\hline $\begin{array}{l}\text { Helicteres baruensis Jacq. } \\
\text { JM } 2298\end{array}$ & & $x$ & & & & & & \\
\hline $\begin{array}{l}\text { Melochia nodiflora Sw. } \\
\text { EAPG } 776,910\end{array}$ & & $x$ & & & $x$ & & & \\
\hline $\begin{array}{l}\text { Melochia pyramidata L. } \\
\text { CGH } 2332\end{array}$ & & & & & $x$ & & & \\
\hline $\begin{array}{l}\text { Melochia tomentosa L. } \\
\text { CGH 1541, EAPG 813, 1133, } 1406\end{array}$ & $x$ & $x$ & & $x$ & & & & $x$ \\
\hline $\begin{array}{l}\text { Waltheria conzattii Standl. } \\
\text { EAPG 1449, EAPG } 1602\end{array}$ & $\mathrm{X}$ & & $\mathrm{X}$ & & & & & \\
\hline $\begin{array}{l}\text { Waltheria indica L. } \\
\text { EAPG } 1378\end{array}$ & & & & & & & & \\
\hline THEOPHRASTACEAE & & & & & & & & \\
\hline $\begin{array}{l}\text { * Jacquinia macrocarpa Cav. } \\
\text { EAPG } 1883\end{array}$ & & & & & $x$ & & & \\
\hline TILIACEAE & & & & & & & & \\
\hline $\begin{array}{l}\text { Heliocarpus pallidus Rose } \\
\text { CGH 1641, JM 1860, EAPG 822, 871, } \\
1621\end{array}$ & & $x$ & & $x$ & & & & \\
\hline
\end{tabular}


Acta Botanica Mexicana (2001), 56: 19-88

Apéndice. Continuación.

\begin{tabular}{|c|c|c|c|c|c|c|c|c|}
\hline \multirow[t]{2}{*}{ Familias, Especies y Colectas } & \multicolumn{8}{|c|}{ Tipo de vegetación } \\
\hline & SAB & SBC & ME & $\mathrm{MX}$ & SM & $B G$ & VA & VS \\
\hline Luehea candida (Moc. et Sessé ex DC.) & & & & & & & & \\
\hline $\begin{array}{l}\text { Mart. } \\
\text { JM 1818, } 2301\end{array}$ & & $x$ & & & $x$ & & & \\
\hline $\begin{array}{l}\text { Triumfetta falcifera Rose } \\
\text { EAPG 1607, } 1632\end{array}$ & & & $x$ & & & & & \\
\hline TURNERACEAE & & & & & & & & \\
\hline $\begin{array}{l}\text { Turnera diffusa Willd. ex Schult. } \\
\text { CGH 1938, LLO 32, EAPG 1058, 1316, } \\
1395\end{array}$ & $x$ & $\mathrm{x}$ & & & & & & \\
\hline $\begin{array}{l}\text { Turnera ulmifolia L. } \\
\text { EAPG } 1579\end{array}$ & $\mathrm{X}$ & & & & & & & \\
\hline ULMACEAE & & & & & & & & \\
\hline $\begin{array}{l}\text { Celtis iguanaea (Jacq.) Sarg. } \\
\text { CGH 1458, 1563, JM 1901, 2278, } \\
\text { EAPG 864, 923, } 1303\end{array}$ & & $\mathrm{X}$ & & & $\mathrm{X}$ & & & \\
\hline URTICACEAE & & & & & & & & \\
\hline $\begin{array}{l}\text { Pilea microphylla (L.) Liebm. } \\
\text { CGH 1449, } 1473\end{array}$ & & & & $\mathrm{X}$ & & & & \\
\hline $\begin{array}{l}\text { * Pilea serpyllifolia (Poir.) Wedd. } \\
\text { EAPG } 1212\end{array}$ & & & & $\mathrm{X}$ & & & & \\
\hline $\begin{array}{l}\text { Pouzolzia nivea S. Watson } \\
\text { CGH 1427, 1494, 1571, EAPG } 1500\end{array}$ & & $\mathrm{X}$ & & & $\mathrm{X}$ & & & \\
\hline $\begin{array}{l}\text { Urera caracasana (Jacq.) Gaudich. } \\
\text { EAPG } 2015\end{array}$ & & & & & $x$ & & & \\
\hline VERBENACEAE & & & & & & & & \\
\hline $\begin{array}{l}\text { Lantana hirta Graham } \\
\text { ELT } 44\end{array}$ & & & & & & $\mathrm{X}$ & & \\
\hline $\begin{array}{l}\text { * Lippia nodiflora (L.) Michx. } \\
\text { EAPG } 1450\end{array}$ & & & & & & & & $\mathrm{x}$ \\
\hline $\begin{array}{l}\text { Lippia nutans B. L. Rob. et Greenm. } \\
\text { EAPG } 1629\end{array}$ & & $\mathrm{X}$ & & & & & & \\
\hline $\begin{array}{l}\text { Petrea volubilis L. } \\
\text { EAPG } 1040\end{array}$ & & $\mathrm{X}$ & & & & & & \\
\hline $\begin{array}{l}\text { Priva lappulacea (L.) Pers. } \\
\text { CGH } 1483\end{array}$ & & $\mathrm{X}$ & & & & & & \\
\hline
\end{tabular}


Apéndice. Continuación.

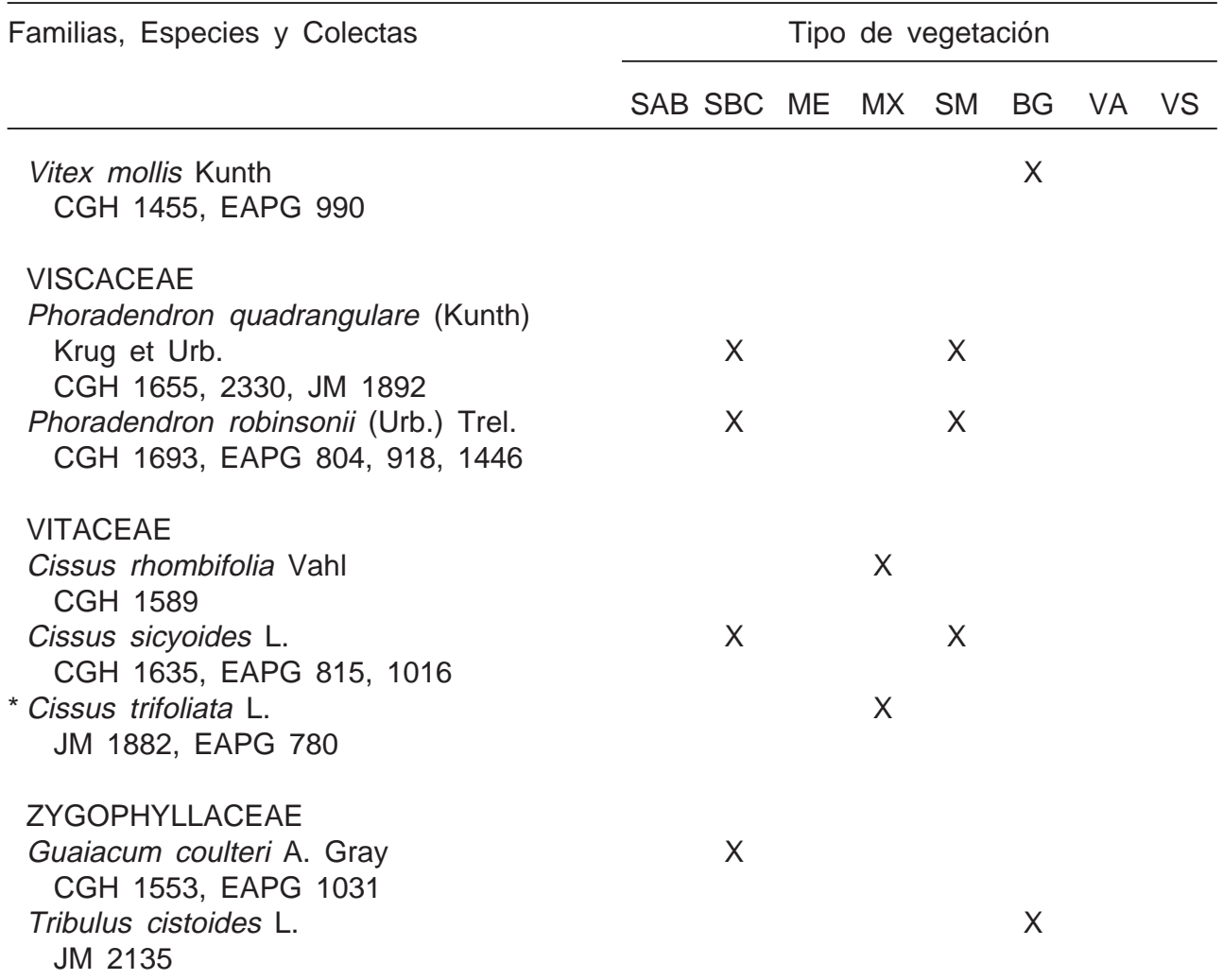

\section{LILIOPSIDA}

AGAVACEAE

Agave angustifolia Haw. $X$

EAPG 945, 1623

Agave ghiesbreghtii Lem. ex Jacobi

EAPG 1026

* Agave nizandensis Cutak

CGH 1428, 1514, JM 1772, EAPG

1817

Manfreda pubescens (Regel et Ortgies)

Verh.-Will. ex Piña

EAPG 1563 
Acta Botanica Mexicana (2001), 56: 19-88

Apéndice. Continuación.

Familias, Especies y Colectas

Tipo de vegetación

SAB SBC ME MX SM BG VA VS

Yucca sp. nov. ined.

EAPG 1864

ALLIACEAE

* Milla oaxacana Ravenna

CGH 1929, 2281, LLO 55, JM 1943

X $\quad x$

AMARYLLIDACEAE

* Zephyranthes nelsonii Greenm.

JM 2154, EAPG 1146, 1935

$X$

ANTHERICACEAE

Echeandia breedlovei Cruden

EAPG 775, 1577, 1585

ARACEAE

$\left(^{*}\right)$ Anthurium cerrobaulense Matuda EAPG 1270

* Anthurium nizandense Matuda CGH 1425, JM 1756, EAPG 1386

$X$

* Philodendron hederaceum (Jacq.) Schott CGH 1924

Philodendron warszewiczii K. Koch et C. D. Bouché EAPG 1172

Syngonium neglectum Schott JM 2102

* Xanthosoma robustum Schott EAPG 1004

ARECACEAE

* Chamaedorea graminifolia H. Wendl.

EAPG 1426, 1427

Sabal mexicana Mart.

EAPG 1245

BROMELIACEAE

Billbergia pallidiflora Liemb. X X JM 1867 
Apéndice. Continuación.

\begin{tabular}{|c|c|c|c|c|c|c|c|}
\hline \multirow[t]{2}{*}{ Familias, Especies y Colectas } & \multicolumn{7}{|c|}{ Tipo de vegetación } \\
\hline & SAB SBC & ME & $\mathrm{MX}$ & SM & $B G$ & VA & VS \\
\hline $\begin{array}{l}\text { Bromelia palmeri Mez } \\
\text { CGH 1463, 1488, } 1513\end{array}$ & $x$ & & & & & & \\
\hline $\begin{array}{l}\text { * Catopsis morreniana Mez } \\
\text { EAPG } 1263\end{array}$ & & & $x$ & & & & \\
\hline $\begin{array}{l}\text { Hechtia caudata L. B. Sm. } \\
\text { CGH 1490, JM } 1766\end{array}$ & & & $x$ & & & & \\
\hline $\begin{array}{l}\text { Hechtia rosea E. Morren ex Baker } \\
\text { JM 1906, EAPG 721, 1227, } 1293\end{array}$ & $x$ & & $\mathrm{x}$ & & & & \\
\hline $\begin{array}{l}\text { * Tillandsia butzii Mez } \\
\text { EAPG } 1264\end{array}$ & & & $\mathrm{x}$ & & & & \\
\hline $\begin{array}{l}\text { Tillandsia caput-medusae E. Morren } \\
\text { JM } 1778\end{array}$ & $\mathrm{x}$ & & & & & & \\
\hline $\begin{array}{l}\text { Tillandsia concolor L. B. Sm. } \\
\text { JM 1762, } 1779\end{array}$ & $x$ & & $x$ & & & & \\
\hline $\begin{array}{l}\text { * Tillandsia drepanoclada Baker } \\
\text { JM 1767, } 1866\end{array}$ & $\mathrm{x}$ & & $\mathrm{x}$ & & & & \\
\hline $\begin{array}{l}\text { * Tillandsia macdougallii L. B. Sm. } \\
\text { EAPG } 869\end{array}$ & $\mathrm{x}$ & & $\mathrm{x}$ & & & & \\
\hline $\begin{array}{l}\text { Tillandsia recurvata (L.) L. } \\
\text { JM 1848, EAPG } 1277\end{array}$ & $\mathrm{x}$ & & $x$ & & & & \\
\hline $\begin{array}{l}\text { Tillandsia schiedeana Steud. } \\
\text { JM 1777, } 1847\end{array}$ & $x$ & & $x$ & & & & \\
\hline $\begin{array}{l}\text { Tillandsia setacea Sw. } \\
\text { CGH } 1475\end{array}$ & $\mathrm{x}$ & & & & & & \\
\hline COMMELINACEAE & & & & & & & \\
\hline $\begin{array}{l}\text { Callisia gentlei Matuda } \\
\text { JM 1933, EAPG 779, } 1554\end{array}$ & $\mathrm{x}$ & & & & & & \\
\hline $\begin{array}{l}\text { * Callisia multiflora (M. Martens et Galeotti) } \\
\text { Standl. } \\
\text { CGH 2339, EAPG 765, } 847\end{array}$ & $\mathrm{x}$ & & & & & & \\
\hline $\begin{array}{l}\text { * Commelina diffusa Burm. f. } \\
\text { EAPG } 1372\end{array}$ & $\mathrm{x}$ & & & & & & \\
\hline $\begin{array}{l}\text { * Commelina rufipes Seub. var. glabrata } \\
\text { (D. R. Hunt.) Faden et D. R. Hunt } \\
\text { CGH 1496, JM 1855, EAPG 1312, } \\
1553\end{array}$ & $\mathrm{x}$ & & $\mathrm{x}$ & & & & \\
\hline Tradescantia andrieuxii C. B. Clarke & $x$ & & & & & & \\
\hline
\end{tabular}


Acta Botanica Mexicana (2001), 56: 19-88

Apéndice. Continuación.

Familias, Especies y Colectas

Tipo de vegetación

SAB SBC ME MX SM BG VA VS

CYPERACEAE

* Abildgaardia ovata (Burm. f.) Kral X CGH 2293, JM 2331

* Bulbostylis capillaris (L.) C. B. Clarke X CGH 2310

Bulbostylis juncoides (Valhl) Kük. ex Osten

JM 2400

* Bulbostylis paradoxa (Spreng.) Lindm. X LLO 91

* Bulbostylis vestita (Kunth) C. B. Clarke X CGH 2291

* Cyperus aggregatus (Willd.) Endl. LLO 70, JM 2304, EAPG 1787

Cyperus canus J. C. Presl et C. Presl CGH 1623, EAPG 924

* Cyperus ciliatus Jungh. CGH 2294, 2303

Cyperus compressus L. EAPG 1825

* Cyperus iria L. LLO 75

* Cyperus odoratus L. EAPG 1835

* Cyperus rotundus L. EAPG 1182, 1526

$x$

$\mathrm{X}$

$X$

X $\mathrm{X}$

$X$

$X$

$X$

$X$

$X$

$X$

X $\quad x$

$\mathrm{X}$

$X$

$\mathrm{X}$

* Fimbristylis spadicea (L.) Vahl CGH 1927, EAPG 1609

* Rhynchospora colorata (L.) H. Pfeiffer X AOP 208, JM 2110

DIOSCOREACEAE

Dioscorea carpomaculata Téllez et B. G.

Schub.

X

EAPG 1803 
Apéndice. Continuación.

Familias, Especies y Colectas

Tipo de vegetación

SAB SBC ME MX SM BG VA VS

Dioscorea convolvulacea Schltld. et Cham. JM 1881

Dioscorea densiflora Hemsl. CGH 2315, JM 1844

Dioscorea floribunda M. Martens et Galeotti CGH 1581

* Dioscorea jaliscana S. Watson EAPG 1549

Dioscorea mexicana Scheidw. CGH 1594, JM 1775, EAPG 1337

* Dioscorea preslii Steud. CGH 1486

$x$

$X$

$X$

$X$

$X$

$X$

HYPOXIDACEAE

* Curculigo scorzonerifolia (Lam.) Baker X JM 2109

\section{IRIDACEAE}

* Alophia drummondii (Graham) R. C. Foster

LLO 69, EAPG 1518

LEMNACEAE

* Lemna valdiviana Phil. EAPG 1654

MARANTACEAE

Maranta arundinacea L.

EAPG 1419

NOLINACEAE

* Beaucarnea sp. nov. ined. JM 1935

$X \quad X$

ORCHIDACEAE

* Barkeria whartoniana (C. Schweinf.) Soto Arenas

EAPG 823, 830, 831, 832, 891, 892, 893, 894, 895, 896, 1459, 1622 
Acta Botanica Mexicana (2001), 56: 19-88

Apéndice. Continuación.

Familias, Especies y Colectas

Tipo de vegetación

SAB SBC ME MX SM BG VA VS

* Bletia coccinea La Llave et Lex. EAPG 1551, 1778

* Bletia purpurea (Lam.) DC. AOP 211

* Cattleya aurantiaca (Bateman) P. N. Don EAPG 1333

Clowesia dodsoniana E. Aguirre JM 1931, EAPG 1127, 1170

Cohniella cebolleta (Jacq.) Christenson EAPG 824, 841, 848, 849, 852, 889, 1383

Cyrtopodium paniculatum (Ruiz et Pav.) Garay

CGH 1586, 1983, EAPG 1070, 1086, 1460

* Encyclia hanburii (Lindl.) Schltr. EAPG 980, 1130, 1168, 1169, 1443

* Encyclia hanburii $x$ nizandensis EAPG 981, 982, 1288

* Encyclia parviflora Regel CGH 1445, EAPG 1458, 1761

* Encyclia sp. nov. ined.

JM 1759, 1773, EAPG 950, 1129, 1501

* Epidendrum ciliare L. EAPG 1750

* Habenaria trifida Kunth X JM 2409

* Lophiaris oerstedii (Rchb. f.) Dressler X EAPG 1800

* Maxillaria tenuifolia Lindl. $\mathrm{X}$ EAPG 1801

* Mesadenus lucayanus (Britton) Schltr. X CGH 1705, EAPG 947

Myrmecophila aff. tibicinis (Bateman) Rolfe EAPG 1452, 1453, 1454, 1455, 1456, 1457

Notylia orbicularis A. Rich. et Galeotti EAPG 1392

$X$

$X$

$X$

$X \quad X$

$X$

$X$

$X$

$\mathrm{X} \quad \mathrm{X}$

$X$

$x$

$X$

$x$ 
Apéndice. Continuación.

Familias, Especies y Colectas

Tipo de vegetación

SAB SBC ME MX SM BG VA VS

* Pleurothallis digitale Luer EAPG 1790

* Prosthechea livida (Lindl.) W. E. Higgins EAPG 1336

Sacoila lanceolata (Aubl.) Garay EAPG 1943

Sarcoglottis assurgens (Rchb. f.) Schltr. EAPG 1774

* Triphora gentianoides (Spreng.) Ames et Schltr.

$X$

EAPG 1484

* Triphora aff. trianthophora (Sw.) Rydb. CGH 1920

POACEAE

*Andropogon fastigiatus Sw. X EAPG 1594

* Andropogon pringlei Scribn. et Merr. X EAPG 1063

Aristida adscensionis L. CGH 2306, EAPG 1597

* Aristida aff. scribneriana Hitchc. X JM 2410

* Aristida jorullensis Kunth X EAPG 1523

* Aristida roemeriana Scheele X JM 2403

* Aristida ternipes Cav. var. ternipes $\mathrm{X}$ EAPG 1184, 1529

Bouteloua chondrosioides (Kunth) Benth. ex S. Watson

CGH 1928, 2270

Bouteloua curtipendula (Michx.) Torr. X CGH 2288

Bouteloua hirsuta Lag. X EAPG 1065

* Bouteloua repens (Kunth) Scribner et Merr.

X

$X$

X

X

$x$

X

$x$

x

$x$

$x$

EAPG 1508 
Acta Botanica Mexicana (2001), 56: 19-88

Apéndice. Continuación.

Familias, Especies y Colectas

Tipo de vegetación

SAB SBC ME MX SM BG VA VS

* Cenchrus brownii Roem. et Schult.

$X$ CGH 1873

* Cenchrus multiflorus J. C. Presl

$X$ CGH 2296, EAPG 1590

Cenchrus pilosus Kunth

$\mathrm{X}$

EAPG 1248

Dactyloctenium aegyptium (L.) Willd.

CGH 1852

Digitaria bicornis (Lam.) Roem. et Schult. CGH 1850

* Digitaria hitchcockii (Chase) Stuck. X LLO 99

Digitaria insularis (L.) Fedde EAPG 1183, 1224

Echinochloa colona (L.) Link EAPG 1181, 1250, 1616

Eragrostis cilianensis (All.) Link ex Vignolo EAPG 1188

Eragrostis ciliaris (L.) R. Br. var. ciliaris EAPG 1538

* Eragrostis hondurensis R. W. Pohl EAPG 821

Guadua amplexifolia J. Presl EAPG 2036

Hackelochloa granularis (L.) Kuntze CGH 2298, EAPG 1778

Heteropogon contortus (L.) P. Beauv. ex Roem. et Schult. EAPG 1179, 1595

* Hyparrhenia rufa (Nees) Stapf X EAPG 1596

Lasiacis grisebachii (Nash) Hitchc. var. grisebachii JM 1782 1897, EAPG 865

Leptochloa mucronata (Michx.) Kunth X X EAPG 1612

Muhlenbergia emersleyi Vasey

EAPG 1254 x 
Apéndice. Continuación.

Familias, Especies y Colectas

Tipo de vegetación

SAB SBC ME MX SM BG VA VS

* Olyra latifolia L.

$\mathrm{X}$

ELT 27

Oplismenus burmannii (Retz.) P. Beauv.

var. burmannii

$X$

$x$

EAPG 769, 1615

Panicum parcum Hitchc. et Chase X EAPG 1521

Panicum trichoides Sw.

EAPG 770

* Panicum tuerckheimii Hack.

$X$ EAPG 1611

* Paspalum centrale Chase X LLO 93

* Paspalum pectinatum Nees X JM 2389

* Paspalum pubiflorum Rupr. ex E. Fourn. X CGH 2301

Rhynchelytrum repens (Willd.) C. E. Hubb.

JM 1849

* Rottboellia cochinchinensis (Lour.) Clayton EAPG 1180

* Schizachyrium brevifolium (Sw.) Nees ex Buse EAPG 1561, 1574

* Schizachyrium cirratum (Hack.) Wooton et Standl. EAPG 1522

* Schizachyrium aff. cirratum (Hack.) Woot. et Standl. JM 2399

* Schizachyrium sanguineum (Retz.) Alston X CGH 2307, EAPG 1572, 1598

Setaria grisebachii E. Fourn.

CGH 1579, EAPG 1187

Sorghum halepense (L.) Pers.

$\mathrm{X}$

EAPG 1186, 1610

* Sporobolus pyramidatus (Lamb.) A. Hitchc.

X

CGH 1851 
Acta Botanica Mexicana (2001), 56: 19-88

Apéndice. Continuación.

Familias, Especies y Colectas

* Thrasya robusta Hitchc. et Chase CGH 2309

Trachypogon plumosus (Humb. et Bonpl. ex Willd.) Nees

EAPG 1064

Tragus berteronianus Schult.

EAPG 1251

* Tripsacum lanceolatum Rupr. ex Benth.

JM 2361

Urochloa fasciculata (Sw.) R. D. Webster CGH 1849

Urochloa mollis (Sw.) Morrone et Zuloaga $\mathrm{X}$ EAPG 1777

SMILACACEAE

* Smilax spinosa Mill. EAPG 1467

TYPHACEAE

Typha domingensis Pers. EAPG 1243
Tipo de vegetación

SAB SBC ME MX SM BG VA VS

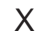

$\mathrm{X}$

$\mathrm{X}$

$X$

88 